\title{
A novel role of sphingosine 1-phosphate receptor S1pr1 in mouse thrombopoiesis
}

\author{
Lin Zhang, ${ }^{1,3}$ Martin Orban,, ${ }^{1,3}$ Michael Lorenz, ${ }^{1,3}$ Verena Barocke, ${ }^{1,3}$ \\ Daniel Braun,,$^{1,3}$ Nicole Urtz, ${ }^{1,3}$ Christian Schulz, ${ }^{1,3}$ \\ Marie-Luise von Brühl, ${ }^{1,3}$ Anca Tirniceriu, ${ }^{1,3}$ Florian Gaertner, ${ }^{1,3}$ \\ Richard L. Proia, ${ }^{4}$ Thomas Graf, ${ }^{5,6}$ Steffen-Sebastian Bolz, ${ }^{7}$ \\ Eloi Montanez, ${ }^{8}$ Marco Prinz, ${ }^{9,10}$ Alexandra Müller, ${ }^{9}$ \\ Louisa von Baumgarten, ${ }^{2}$ Andreas Billich, ${ }^{11}$ Michael Sixt,${ }^{8}$ \\ Reinhard Fässler, ${ }^{8}$ Ulrich H. von Andrian, ${ }^{12}$ Tobias Junt, ${ }^{11}$ \\ and Steffen Massberg ${ }^{1,3,13}$
}

\footnotetext{
'Medizinische Klinik und Poliklinik I and ${ }^{2}$ Neurologische Klinik, Klinikum der Universität, Ludwig-Maximilian-Universität München, 81337 Munich, Germany

${ }^{3}$ Deutsches Herzzentrum München, Technische Universität München, 80636 Munich, Germany

${ }^{4}$ Genetics of Development and Disease Branch, National Institute of Diabetes and Digestive and Kidney Diseases, National Institutes of Health, Bethesda, MD 20892

${ }^{5}$ Centre for Genomic Regulation, 08003 Barcelona, Spain

${ }^{6}$ Catalan Institution for Research and Advanced Studies (ICREA), 08010 Barcelona, Spain

${ }^{7}$ Department of Physiology, University of Toronto, Toronto, Ontario, Canada M5S 1A8

${ }^{8}$ Max Planck Institute of Biochemistry, 82152 Martinsried, Germany

9 Pathologisches Institut, Abteilung Neuropathologie, Universitäts Klinikum Freiburg, 79106 Freiburg, Germany

${ }^{10 B I O S S}$ Centre for Biological Signalling, University of Freiburg, 79108 Freiburg, Germany

${ }^{11}$ Novartis Institutes for BioMedical Research, 4002 Basel, Switzerland

${ }^{12}$ Department of Pathology, Harvard Medical School, Boston, MA 02115

${ }^{13}$ Munich Heart Alliance, German Cardiovascular Research Centre, 80802 Munich, Germany
}

Millions of platelets are produced each hour by bone marrow (BM) megakaryocytes (MKs). MKs extend transendothelial proplatelet (PP) extensions into BM sinusoids and shed new platelets into the blood. The mechanisms that control platelet generation remain incompletely understood. Using conditional mutants and intravital multiphoton microscopy, we show here that the lipid mediator sphingosine 1-phosphate (S1P) serves as a critical directional cue guiding the elongation of megakaryocytic PP extensions from the interstitium into BM sinusoids and triggering the subsequent shedding of PPs into the blood. Correspondingly, mice lacking the S1P receptor S1 pr 1 develop severe thrombocytopenia caused by both formation of aberrant extravascular PPs and defective intravascular PP shedding. In contrast, activation of S1 pr 1 signaling leads to the prompt release of new platelets into the circulating blood. Collectively, our findings uncover a novel function of the S1P-S1 pr1 axis as master regulator of efficient thrombopoiesis and might raise new therapeutic options for patients with thrombocytopenia.

\section{CORRESPONDENCE}

Steffen Massberg:

steffen.massberg@

med.uni-muenchen.de

Abbreviations used: 3D, three dimensional; DIC, differential interference contrast; DMS, invaginated demarcation membrane system; FL, fetal liver; MK, megakaryocyte; MP-IVM, multiphoton intravital microscopy; PP, proplatelet; PPF, PP formation; S1P, sphingosine 1-phosphate; TPO, thrombopoietin.
Billions of anucleated platelets circulate in mammalian blood to prevent blood loss in case of tissue injury. The lifespan of platelets is short (4-6 d in mice and 5-9 d in humans; Leeksma and Cohen, 1955; Robinson et al., 2000); as a consequence, several million platelets have to be produced every hour to maintain their physiological blood counts and to avoid the risk of bleeding. In mammals, platelets are generated in BM from megakaryocytes (MKs), polyploid, terminally differentiated myeloid cells with a typical morphology and diameters of up to $100 \mu \mathrm{m}$.
The production of platelets from MKs involves several sequential developmental and maturation steps. MKs develop from hematopoietic stem and progenitor cells, which give rise to an increasingly restricted lineage culminating in the formation of megakaryocytic precursors that generate MKs. During their differentiation and maturation, MKs localize to

2012 Zhang et al. This article is distributed under the terms of an AttributionNoncommercial-Share Alike-No Mirror Sites license for the first six months after the publication date (see http://www.rupress.org/terms). After six months it is available under a Creative Commons License (Attribution-Noncommercial-Share Alike 3.0 Unported license, as described at http://creativecommons.org/licenses/by-nc-sa/3.0/ 
the perivascular niche, where they interact with sinusoidal BM endothelial cells (Avecilla et al., 2004; Patel et al., 2005a). Once they have settled in the perivascular microenvironment, mature MKs form dynamic transendothelial pseudopods, which extend into the lumen of BM sinusoids. These intravascular pseudopodial extensions, termed proplatelets ( $\mathrm{PPs})$, continue to elongate and become tapered into multiple platelet-size beads connected to each other and with their maternal MKs by thin cytoplasmic bridges (Italiano et al., 1999; Patel et al., 2005a). The release of platelets, the final step of platelet formation, then occurs within the blood, where new platelets are shed as fragments from the tips of intravascular PPs (Stenberg and Levin, 1989; Choi et al., 1995; Italiano et al., 1999; Junt et al., 2007).

MKs are a rare cell population, constituting $<0.01 \%$ of all BM cells. This contrasts with the high demand of platelet production, implying that the differentiation of MKs (termed megakaryocytopoiesis) and the subsequent assembly and release of platelets by MKs (termed thrombopoiesis) are highly efficient and tightly controlled processes. Among the factors that modulate megakaryocytopoiesis, thrombopoietin (TPO) is the major regulator of MK expansion from hematopoietic stem and progenitor cells, whereas chemokines, including stromal-derived factor-1 (SDF-1), primarily initiate the relocation of maturing $\mathrm{MKs}$ to the perivascular microenvironment (Avecilla et al., 2004). In contrast, the molecular pathways that control the final steps of thrombopoiesis, particularly the guidance signals that direct megakaryocytic pseudopodial extensions into the vascular lumen and trigger the intravascular release of new platelets, are entirely unknown.

The bioactive sphingolipid sphingosine 1-phosphate (S1P) and the receptors responsive to this mediator regulate important biological functions of various hematopoietic cell types (Spiegel and Milstien, 2003, 2011; Schwab et al., 2005; Massberg et al., 2007), including cell migration in the BM compartment (Ishii et al., 2009; Allende et al., 2010). Here we report that $\mathrm{S} 1 \mathrm{P}$ and the $\mathrm{MK} \mathrm{S} 1 \mathrm{P}$ receptor $\mathrm{S} 1 \mathrm{pr} 1$ receptor are indispensable for normal BM thrombopoiesis. Using mouse mutants and by multiphoton intravital microscopy (MP-IVM), we demonstrate that a transendothelial S1P gradient navigates megakaryocytic PP extensions into the lumen of BM sinusoids. In the blood, PP extensions are exposed to high S1P concentrations, which initiate the subsequent shedding of platelets into the circulation. Both processes involve the S1P receptor S1pr1, triggering activation of the Gi/Rac GTPase signaling. Correspondingly, lack of S1 pr1 on MKs, but not of other S1P receptors, results in severe thrombocytopenia.Thus, we have identified the S1P-S1pr1 pathway as a key nodal point integrating guidance cues that navigate directional PP elongation and enabling the final step of thrombopoiesis, the shedding of new platelets into the blood stream.

\section{RESULTS}

\section{S1pr1 expression in MKs intrinsically regulates} platelet homeostasis

We observed here that cultured mouse and human MKs, as well as the human megakaryocytic cell lines Meg01 and
CMK, each express the S1P receptor subtypes 1,2 , and 4 (encoded by S1pr1 and S1PR1, S1pr2 and S1PR2, and S1pr4 and S1PR 4 in mice and humans, respectively; Fig. 1,A-E; and Table S1). To directly test whether $\mathrm{S} 1 \mathrm{P}$ receptors play a role for megakaryo- or thrombopoiesis, we determined platelet counts in peripheral blood of WT mice and mice lacking the $\mathrm{S} 1 \mathrm{P}$ receptors expressed by MKs. Loss of S1pr2 or S1pr4 on hematopoietic cells had no significant effects on peripheral platelet counts or platelet size (Fig. 1 F, Table S2, and not depicted). In contrast, ablation of the S1pr1 gene was associated with dramatically reduced platelet numbers. Loss of one S1pr1 allele (S1 pr1 ${ }^{+/-}$mutants) already resulted in a significant reduction in the number of circulating platelets (Fig. $1 \mathrm{G}$ and Table S3). Loss of both alleles (S1 pr1 $1^{-/-}$mice) was embryonically lethal (Liu et al., 2000); thus, to circumvent embryonic lethality, we generated chimaeras by transferring fetal liver (FL) cells from S1pr1 $1^{-/-}, \mathrm{S} 1 \mathrm{pr}^{+/-}$, or S1pr1 $1^{+/+}$donors into irradiated WT mice. 6-8 wk after reconstitution, BM cells from S1pr1 $1^{-/-}, \mathrm{S} 1 \mathrm{pr}^{+/-}$, or S1pr1 $1^{+/+} \mathrm{FL}$ chimaeras were isolated and further transplanted into irradiated secondary recipient mice. Platelet counts in $\mathrm{S} 1 \mathrm{pr}^{+/-}$and $\mathrm{S} 1 \mathrm{pr} 1^{-/-}$ chimaeras were reduced by $>50 \%$ and $70 \%$ compared with S1pr1 ${ }^{+/+}$chimaeras, respectively (Fig. $1 \mathrm{G}$ and Table S2). Collectively, these results indicate that S1pr1 on hematopoietic cells controls blood platelet homeostasis, whereas S1pr2 and S1pr4 are dispensable for this process.

Next we evaluated whether S1pr1 expressed by MKs or by other hematopoietic lineages regulates the number of blood platelets. To this end, we reconstituted irradiated mice with BM cells carrying two floxed S1pr1 alleles (S1pr1 $\left.1^{\mathrm{f} / \mathrm{fl}}\right)$ and transduced with a lentivirus expressing Cre recombinase under the MK-specific GpIIb promoter (GpIlb-Cre S1pr1 $1^{\mathrm{fl} / \mathrm{fl}}$ ) to delete $S 1$ pr1 in the $\mathrm{MK} /$ platelet progeny (Fig. $1 \mathrm{H}$; Allende et al., 2003). Importantly, platelet counts became significantly reduced in GpIIb-Cre S1 pr1 $1^{\mathrm{f} / \mathrm{fl}} \mathrm{BM}$ recipients as compared with S1 pr1 $1^{\mathrm{f} / \mathrm{fl}}$ control chimaeras (Fig. $1 \mathrm{H}$ ). Moreover, lentiviral reexpression of S1pr1 under the MK-specific GpIb $\alpha$ promoter rescued S1 pr1 $1^{-/-}$FL cells to reconstitute the blood platelet compartment in lethally irradiated mice (Fig. 1 I). These findings demonstrate that S1pr1 expressed by the MK lineage intrinsically controls platelet homeostasis.

\section{Normal MK development, platelet life span, and serum TPO levels in S1pr1-deficient mice}

What could be the reason for the severe thrombocytopenia in the absence of S1pr1? First we showed that the life spans of platelets from S1 pr1 $1^{+/+}, \mathrm{S} 1 \mathrm{pr}^{+/-}$, or S1 pr1 $1^{-/-}$ chimaeras and between $\mathrm{WT}$ and $\mathrm{S} 1 \mathrm{pr}^{+/-}$mutant mice was similar, excluding a reduced life span as cause for the reduced platelet counts (Fig. $2 \mathrm{~A}$ ). We also excluded a defect in the release of TPO, the principle regulator of thrombopoiesis (Kaushansky, 2005a), as cause for the thrombocytopenia in S1pr1-null mutants (Fig. 2 B). Finally, we also could not find evidence for a gross defect in MK development, as we found similar numbers of megakaryocytic progenitor cells in WT and S1 pr1 $1^{-/-}$FL cells populations 

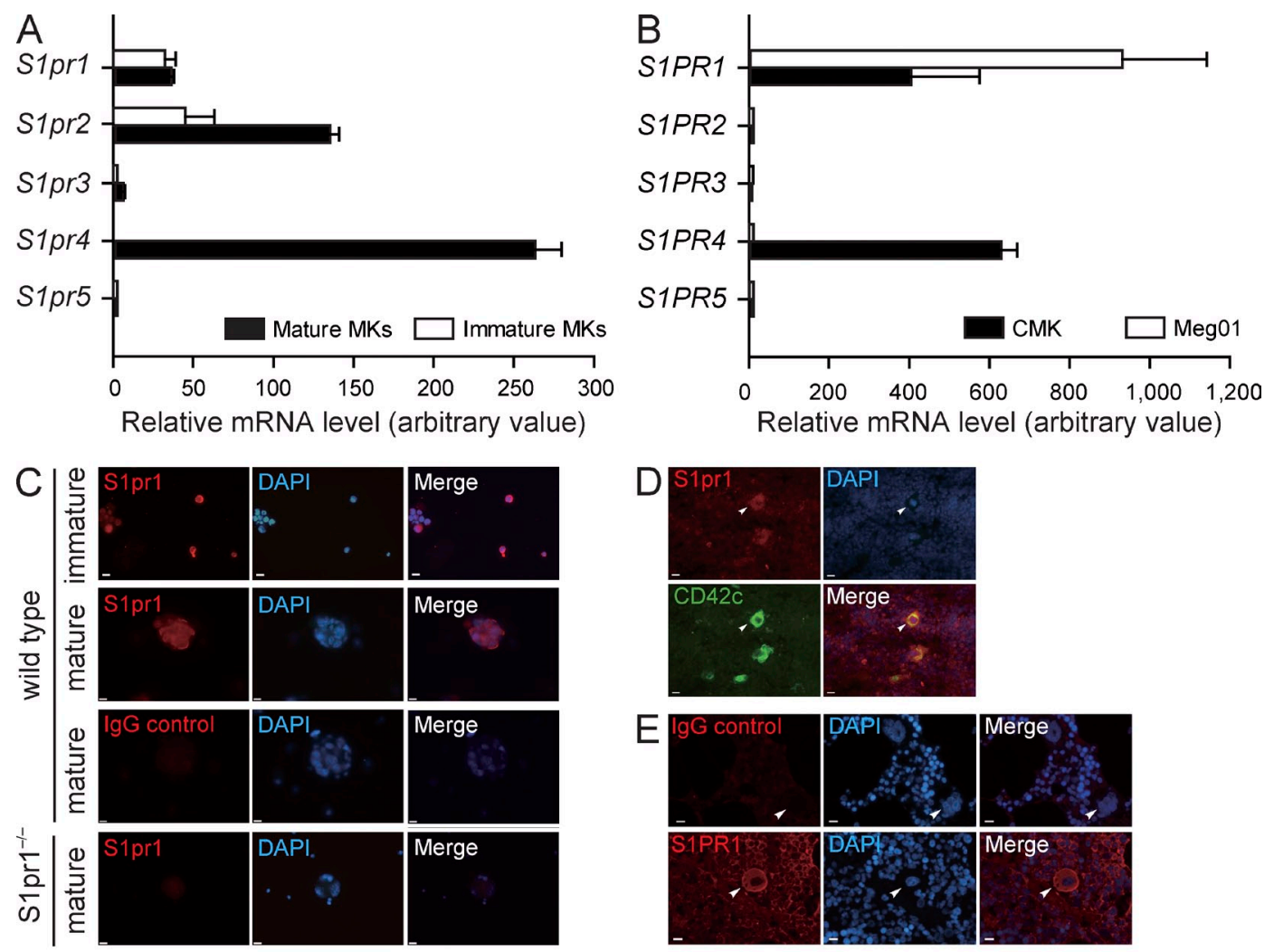

F
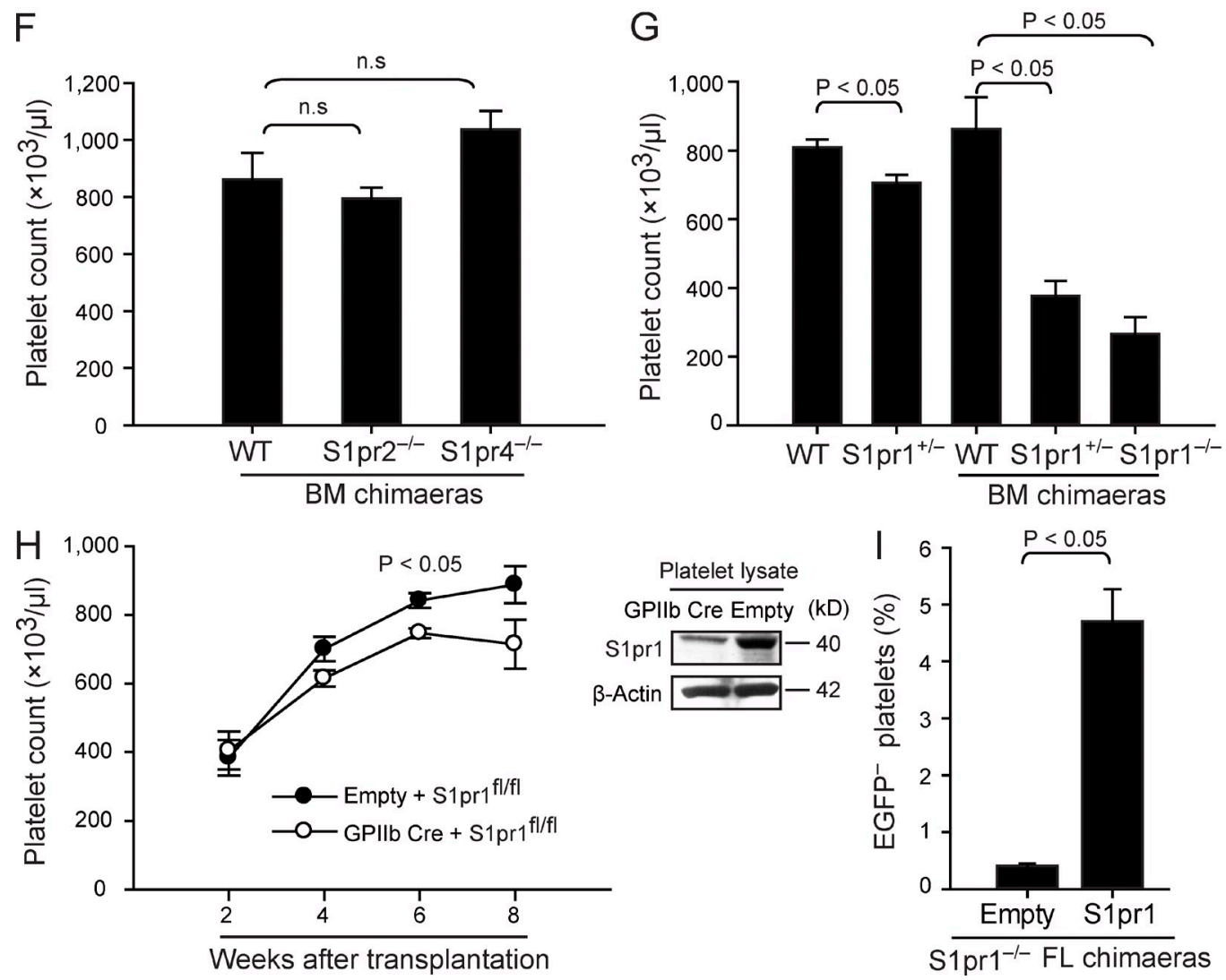

Figure 1. MKs express S1 pr1, and S1 pr1-deficient mice display severe thrombocytopenia. (A) Relative expression of S1P receptor mRNA by FL-derived mature and immature MKs. (B) Relative expression of S1P receptor mRNA in human megakaryocytic cell lines. (A and B) Data are representative of three independent experiments with triplication. (C) Representative immunostaining of S1pr1 in immature and mature FL-derived MKs. WT MKs stained 
(Fig. 2 C) and a normal differentiation of WT and S1 pr1 $1^{-1-}$ precursor cells into MKs both in vitro (Fig. $2 \mathrm{D}$ ) and in vivo (Fig. 2, E and F).

\section{Loss of S1pr1 increases MK size but has no effect on positioning and motility of MKs in vivo}

Next we examined whether S1pr1 controls platelet biogenesis for example by modulating MK motility or their positioning within the BM compartment. To address this question, we performed MP-IVM of calvarian BM (Junt et al., 2007) of two different sets of S1 pr1 $1^{+/+}$or S1 pr1 ${ }^{-/-}$chimaeras, in which $\mathrm{MKs}$ and their progeny were genetically marked: (a) S1 pr1 ${ }^{+/+}$ or S1pr1-/- CD41-YFPki/+ FL chimaeras, in which MKs and platelets express the YFP driven from the endogenous CD41 gene locus (Zhang et al., 2007) and (b) S1pr1 $1^{+/+}$or S1pr1 $1^{-/-}$ lenti-GpIb $\alpha$-enhanced GFP (EGFP) BM chimaeras, in which MKs and platelets express EGFP under the transcriptional control of the murine GpIb $\alpha$ promoter (Lavenu-Bombled et al., 2007). The experiments revealed neither differences in MK size nor in their positioning or motility when we compared S1pr1 ${ }^{+/+}$CD41-YFPki/+ or S1pr1 ${ }^{+/+}$lenti-GpIb $\alpha-E G F P$ chimaeras and naive (nontransplanted) $\mathrm{S} 1 \mathrm{pr}^{+/+} \mathrm{CD} 41-\mathrm{YFP}^{\mathrm{ki} /+}$ or platelet factor 4 (Pf4)-EYFP transgenic mice, in which EYFP is driven by the MK-specific Pf4 promoter, which allowed excluding a major influence of irradiation and BM transplantation (Fig. 3,A-D). As reported previously (Junt et al., 2007), S1 pr1 ${ }^{+/+}$MKs were large, mostly sessile cells always located in close proximity to BM sinusoids (Fig. 3, A and D-G). In S1pr1 $1^{-/-}$chimaeras and S1pr1 ${ }^{+/-}$mice, MKs were significantly larger compared with S1 pr1 ${ }^{+/+}$chimaeras, whereas the position and motility of MKs was similar among all genotypes (Fig. 3, E-G). The aforementioned results suggest that in contrast to other cells in the BM (Ishii et al., 2009), neither positioning nor migration of MKs or their committed progenitors in marrow spaces is controlled by S1pr1.

\section{S1pr1 is essential for intravascular PP formation (PPF)}

During thrombopoiesis, mature MKs extend transendothelial protrusions, termed PPs, into BM microvessels (Junt et al., 2007). To test whether S1P/S1P receptor signaling plays a role during PPF, we cultured MKs in vitro (Lecine et al., 1998) and found that on average, 9 out of 100 WT MKs spontaneously formed PPs as assessed by phase-contrast microscopy. MKs isolated from S1 pr2 $2^{-/-}$and S1 $\mathrm{pr}^{-/-}$mice generated similar number of PPs (unpublished data). In sharp contrast, in vitro $\mathrm{PPF}$ was reduced by $>70 \%$ in $\mathrm{S} 1 \mathrm{pr} 1^{-/-} \mathrm{MKs}$, as $<2$ out of $100 \mathrm{~S} 1 \mathrm{pr}^{-/-}$MKs formed PPs (Fig. 4 A). Importantly, lentiviral reexpression of GpIb $\alpha$ promoter-driven S1pr1 in S1pr1 $1^{-/-}$MKs corrected PPF in vitro (Fig. 4 B). These results clearly indicate that S1 pr1 plays a critical and intrinsic role for PPF by MKs.

When we examined how S1pr1 might control PPF, we could exclude a primary lack of the invaginated demarcation membrane system (DMS), the predominant reservoir for PP membranes (Radley and Haller, 1982; Schulze et al., 2006), in S1pr1 ${ }^{-/-} \mathrm{MKs}$, as electron microscopy of S1 pr1 $1^{-/-}$ BM MKs did not reveal abnormalities of the DMS when compared with S1pr1 ${ }^{+/+}$BM MKs (Fig. 4 C). Next we tested whether S1P serves as a chemoattractant for polarizing MKs and for inducing the formation of PP protrusions. Within the normal BM compartment, S1P is rapidly degraded by lyases and phosphatases expressed by most hematopoietic cells. Thus, the local S1P concentrations in the BM (with its densely packed hematopoietic cells) are exceedingly low (unpublished data), reflecting similar concentrations reported for other tissues such as lymph nodes (Schwab et al., 2005; Pappu et al., 2007). In contrast, high S1P concentrations exist in the blood stream (Caligan et al., 2000; Berdyshev et al., 2005; Pappu et al., 2007). Because of their positioning at the vascular interface, MKs are therefore exposed to a steep transendothelial $\mathrm{S} 1 \mathrm{P}$ gradient. To mimic the situation in the BM, we exposed cultured MKs to a gradient of S1P in vitro. Notably, PP extensions developed preferentially toward increasing concentrations of S1P but not of vehicle (Fig. 4 D). A similar result was also obtained with $\mathrm{S} 1 \mathrm{pr} 2^{-/-}$and $\mathrm{S} 1 \mathrm{pr}^{-/-} \mathrm{MKs}$ (Fig. 4 D).VPC23019, a previously described S1pr1 and S1pr3 antagonist (Davis et al., 2005), was used in our study to selectively block megakaryocytic S1pr1 signaling because MKs do not express S1pr3 (Fig. 1 A). Inhibition of the megakaryocytic S1pr1 using VPC23019 abolished this directionality of PPF; MKs projected PP extensions into random directions (Fig. 4 D). These findings suggest that S1P-S1pr1 signaling is essential for PPF by providing a chemoattractant stimulus that controls the polarization of $\mathrm{PP}$ processes generated by MKs in culture.

Next we defined the signaling downstream of S1pr1 involved in the regulation of PPF and polarization. Because Rac GTPase activity controls actin dynamics leading to membrane protrusion/extensions (Aspenström et al., 2004), we tested whether activation of Rac GTPases via

with irrelevant control IgG or anti-S1pr1-stained S1pr1-null MKs served as controls. (D and E) Expression of S1pr1 in murine BM (D) and S1PR1 in human BM sections (E). Arrowheads indicate MKs. CD42c is the marker for MKs. All MKs examined stained positive for S1pr1 or S1PR1. Bars, 10 $\mu$ m. (F) Platelet counts in peripheral blood. $n=15$ for WT BM chimaeras; $n=5$ for S1 pr2 ${ }^{-1-}$ BM chimaeras; $n=3$ for S1 pr4 ${ }^{-1-}$ BM chimaeras. (G) Platelet counts in peripheral blood. $n=8$ for WT; $n=6$ for S1 pr $1^{+1-} ; n=15$ for WT BM chimaeras; $n=9$ for S1 pr $1^{+1-}$ BM chimaeras; $n=14$ for S1 pr $1^{-1-}$ BM chimaeras. (H) Platelet counts in chimaeras after transferring S1 pr $1^{\mathrm{fl} / \mathrm{fl}} \mathrm{BM}$ cells transduced with lenti-GPllb-cre or empty control vectors into irradiated recipient mice (left). Expression of S1 pr1 in platelets in chimaeras after transferring S1 pr ${ }^{\text {fl/fl }}$ BM cells transduced with lenti-GPIIb-cre or empty control vectors. $\beta$-Actin served as loading control (right). $n=3$ per genotype. (I) Percentage of EGFP- platelets in chimaeras after hematopoietic reconstitution of lethally irradiated mice with EGFP+ S1 pr1 $1^{+/+}$BM cells and EGFP- S1 pr1 ${ }^{-1-}$ FL cells at a ratio of 20:1. The EGFP- S1 pr1 $1^{-1-}$ FL cells were transduced with lentiGplb $\alpha-S 1$ pr 1 or empty control vectors before transplantation. $n=3$ per genotype. All error bars indicate SEM. 

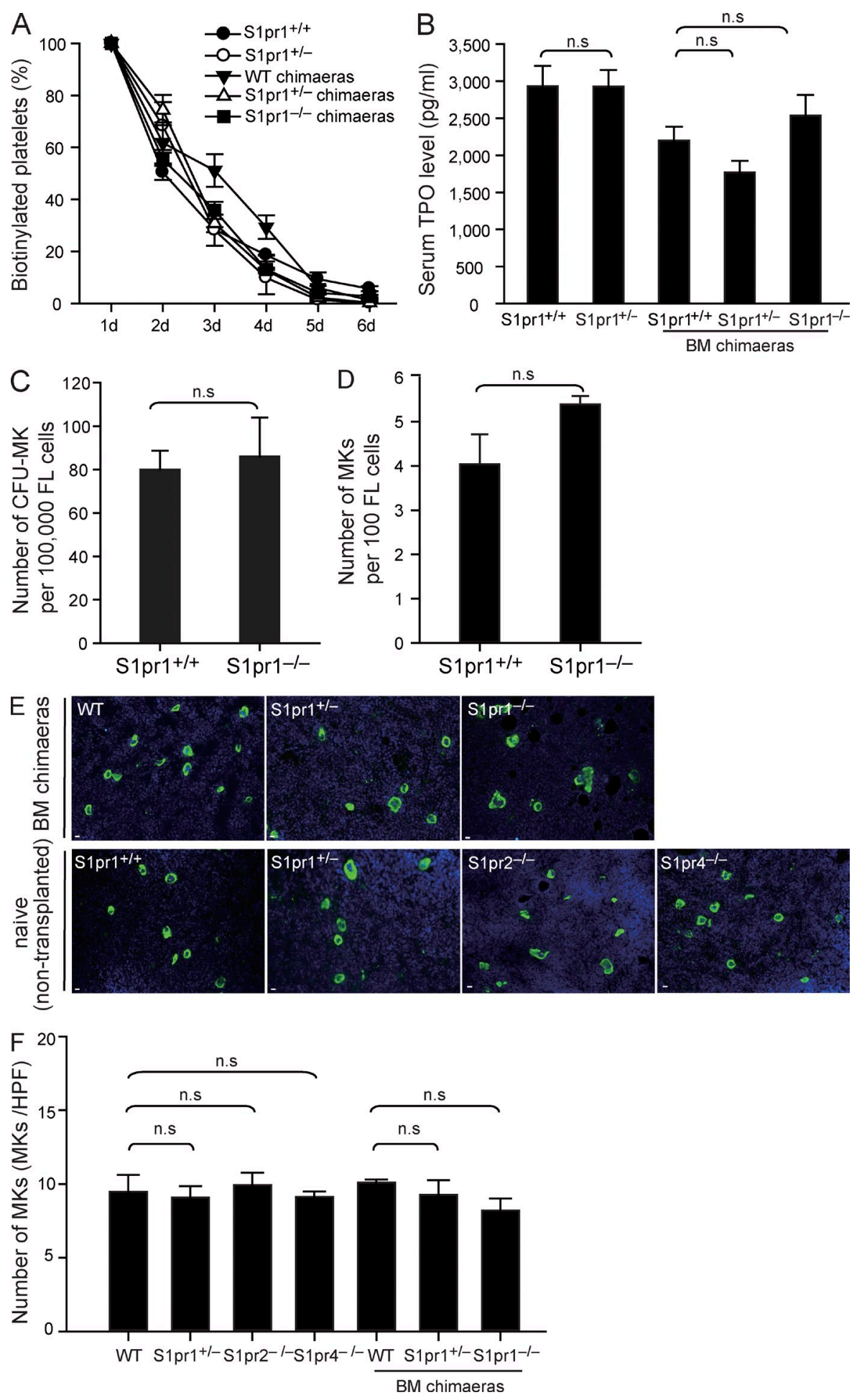

S1pr1 in MKs controls PPF. As reported previously for endothelial cells (Paik et al., 2004), we found that S1P, and also the S1pr1-specific agonist SEW2871, enhances Rac GTPase activity via S1pr1 in megakaryocytic cell lines (Fig. 4, E and F). Conversely, we observed that pharmacological inhibition of Rac GTPases by NSC23766 completely abolished PPF in response to S1P in vitro (Fig. 4 G),
Figure 2. Loss of $\mathrm{S} 1 \mathrm{pr} 1$ does not change platelet life span, serum TPO levels, and MK development. (A) Platelet life span assays in the indicated genotypes. $n=3-5$ per genotype. (B) Serum TPO levels. $n=3-5$ per genotype. (C) Quantification of CFU-MK numbers in FL cells. Data are representative of three independent experiments with triplication. (D) Number of mature MKs in cultured FL cells. Data are representative of four independent experiments with triplication. (E) Representative immunostaining of MKs in mouse femoral BM. MKs were detected by the MKspecific marker CD41 (green). DAPI is stained blue. Bars, $10 \mu \mathrm{m}$. (F) Quantification of MK numbers per 20x high-power field in femoral BM. $n=3$ mice per genotype. All error bars represent SEM. indicating that activation of Rac GTPases downstream of S1pr1 is required for PPF.

To evaluate the in vivo relevance of $\mathrm{S} 1 \mathrm{P}$ and its receptors for PPF, we examined CD41-YFPki/+ mice by MP-IVM. In CD $41-\mathrm{YFP}^{\mathrm{ki} /+}$ mice, $59 \%$ of all MKs extended plump or long PP protrusions into BM sinusoids (Fig. S1 A andVideo 1), indicating active participation in platelet biogenesis. PP protrusions 
A

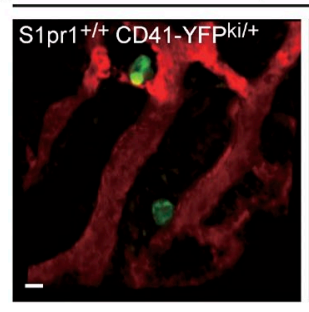

B $\square$ CD41-YFPkil+

$\square$ Pf4-EYFP

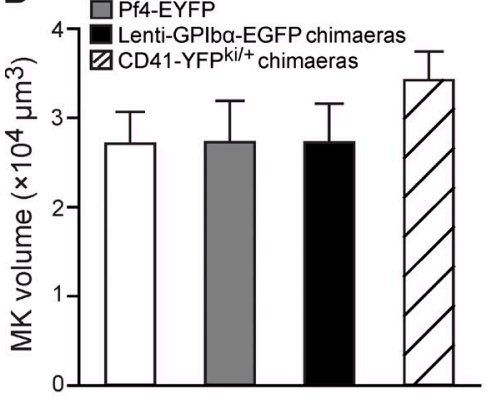

D

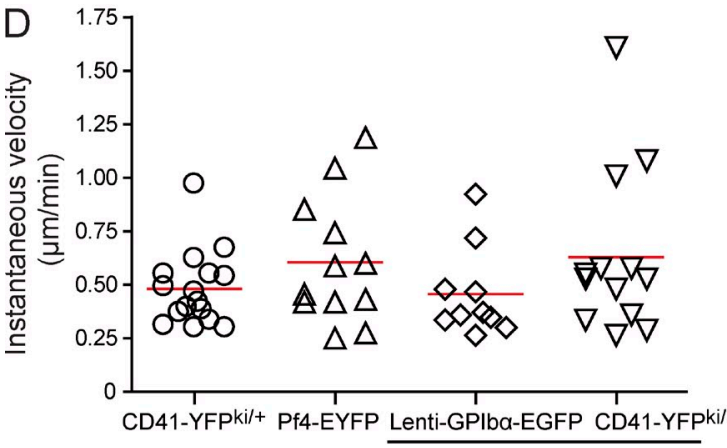

Chimaeras

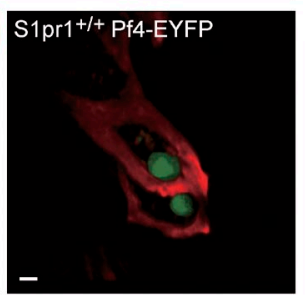

o
Chimaeras
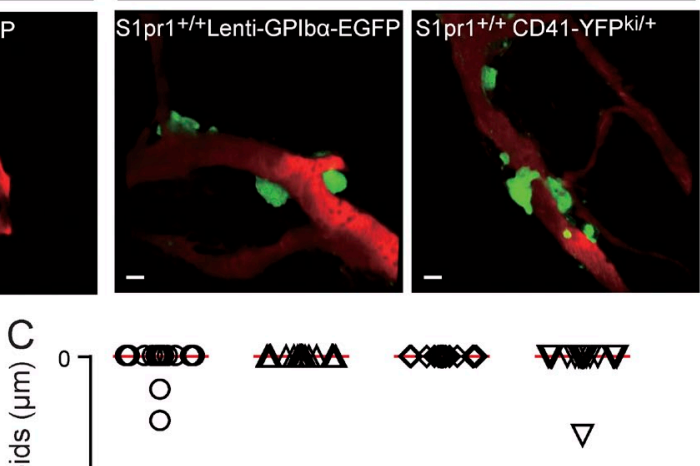

$\diamond$

$\triangle$

CD41-YFPkil+ Pf4-EYFP Lenti-GPlba-EGFP CD41-YFPki/+

Chimaeras
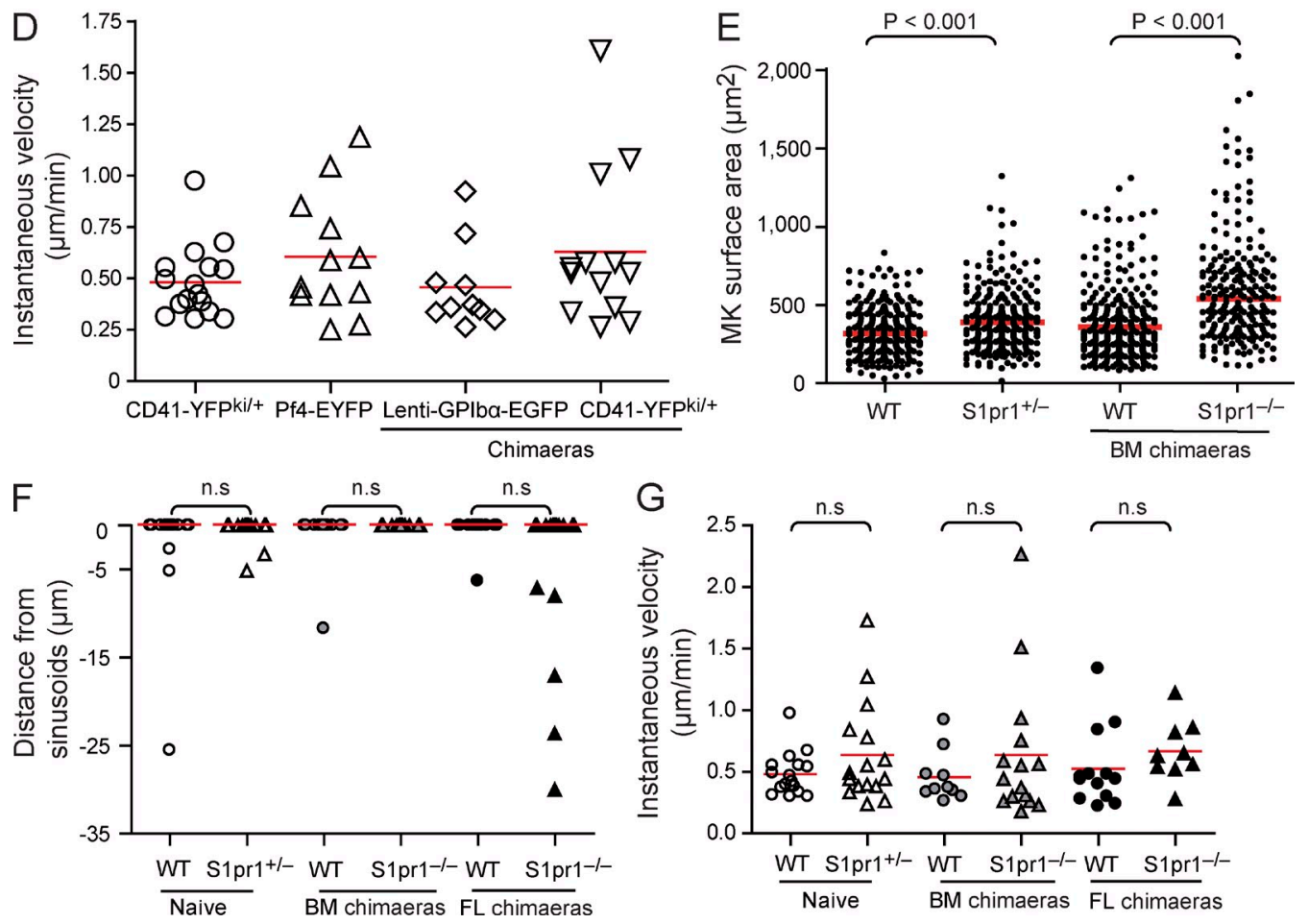

Figure 3. Loss of S1pr1 increases the size but has no effect on the positioning and motility of MKs in vivo. (A) Representative MP-IVM images of YFP+ or EGFP+ MKs (green) in BM. BM microvasculature was visualized by intravenous injection of TRITC-dextran (red). Left, naive (nontransplanted)

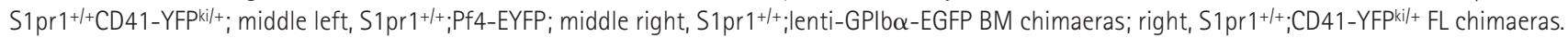
Bars, $20 \mu \mathrm{m}$. (B-D) Volumes (B), distances from sinusoids (C), and the instantaneous lateral ( $x-y)$ velocity (D) of MKs in the indicated groups. Red lines in $C$ indicate medians; red lines in D indicate means. Error bars represent SEM. $n=13-46$ MKs per genotype. Data are pooled from three mice each group. P-values among the different groups in B-D are $>0.05$. (E) Surface area of MKs in BM. (F) Distance of MKs from BM sinusoids. (E and F) Red lines indicate medians. $(G)$ Instantaneous lateral $(x-y)$ velocity of MKs. Red lines indicate means. ( $E-G)$ Data are pooled from three mice each group. 

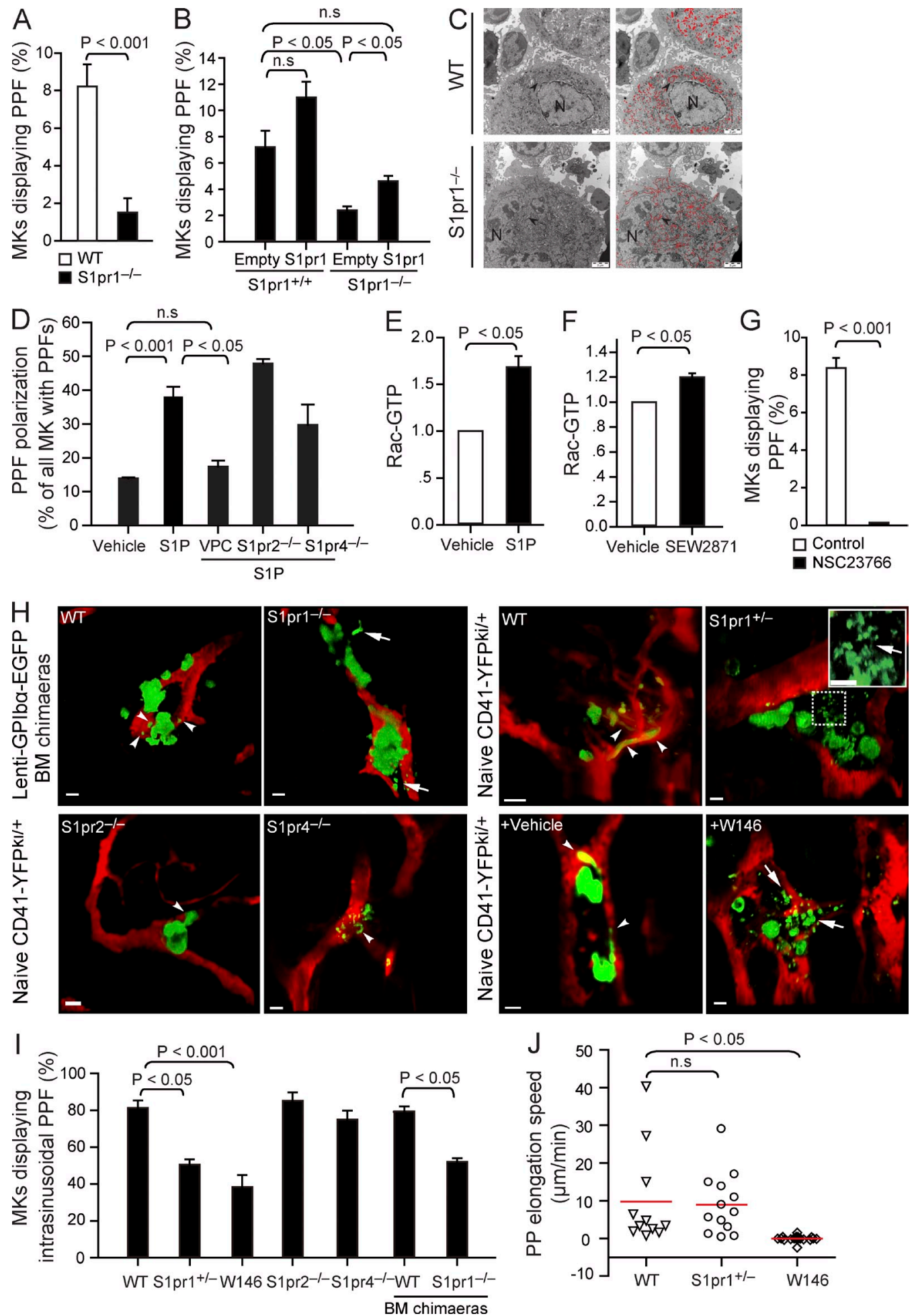

Figure 4. S1P regulates PPF. (A) The percentage of MKs displaying PPF. PPF is expressed as the percentage of MKs carrying PPs (8,000-10,000 MKs per experiment, five independent experiments with triplications). (B) The percentage of MKs displaying PPF in S1pr $1^{+/+}$or S1 pr $1^{-1-}$ MKs transduced with lenti-Gplb $\alpha$-S1pr 1 or empty control vectors (3,000-8,000 MKs per experiment, two independent experiments with triplications). (C) Representative electron micrographs of WT and S1 pr1-1- MKs in BM. Arrowheads indicate the DMS. Red color highlights the DMS. N, nucleus. (D) The percentage of MKs with polarized PPF in the presence or absence of S1P and the S1pr1-specific inhibitor VPC23019 (VPC). $n=127-265$ MKs per group. Data are pooled from three to five independent experiments. (E) Y10/L8057 cells were incubated with $10 \mu \mathrm{M}$ S1P or vehicle for 2 min. The activities of Rac-GTP were quantified by pull-down assay ( $n=5$ independent experiments). (F) Y10/L8057 cells were incubated with $1 \mu \mathrm{M}$ S1pr 1 agonist, SEW2871, or vehicle for 5 min. The activities of Rac-GTP were quantified by pull-down assay ( $n=3$ independent experiments). (G) The percentage of MKs displaying PPF in the presence or 
extended almost exclusively into marrow sinusoids of CD41$\mathrm{YFPki}^{\mathrm{N}+}$ mice, whereas we rarely detected extravascular PP processes (Fig. 4, H and I; andVideo 2). To determine whether the $\mathrm{S} 1 \mathrm{P}$ receptors expressed by $\mathrm{MKs}$ provide the guidance information necessary to direct $\mathrm{PP}$ processes into $\mathrm{BM}$ sinusoids, we examined S1 pr1 ${ }^{+/-}$CD41-YFPki/+, $\mathrm{S} 1 \mathrm{pr} 2^{-/-}$ CD41-YFPki/+ , and S1pr4 ${ }^{-/-} \mathrm{CD} 41-\mathrm{YFP}^{\mathrm{ki} /+}$ mice as well as lenti-GpIb $\alpha$-EGFP BM chimaeras. Consistent with our in vitro findings (Fig. 4 D), loss of S1pr2 or S1pr4 did not affect the formation or polarization of PPs in vivo (Fig. 4, H and I). In contrast, loss of S1 pr1 disrupted PPF and polarization; correspondingly, S1 $\mathrm{pr}^{+/-}$or S1pr1-/- $\mathrm{MKs}$ projected $\mathrm{PP}$ extensions in random directions (Fig. 4, H and I; and Video 2). As a consequence, we found aberrant PP processes in the marrow interstitial space, whereas intrasinusoidal PPs were rarely detected in S1pr1-deficient chimaeras (Fig. 4, H and I; and Video 2). Likewise, when we treated S1 pr1 ${ }^{+/+}$CD41-YFPki/+ mice with the S1pr1-specific antagonist W146 for $24 \mathrm{~h}$, the physiological directionality of PPF was entirely disrupted. We frequently observed long cytoplasmic extensions outside sinusoids in mice treated with W146 but not in vehicle-treated animals (Fig. 4, H and I; and Video 3). In addition, inhibition of S1pr1 also retarded PP growth in vivo, resembling the reduced PPF of cultured S1pr1 ${ }^{-/}$MKs in vitro (Fig. 4, A and J). These results indicate that S1pr1 signaling supports PPF and elongation along the physiological S1P gradient between BM interstitium and BM sinusoids and controls the entry of PPs into the marrow blood stream in vivo.

\section{S1P enhances PP fragmentation via S1 pr1 in vitro}

Once MK PP processes have entered the blood, they are exposed to significantly higher S1P concentrations compared with the BM interstitium (unpublished data). To our surprise, when we mimicked the situation in the blood by incubating cultured MKs to allow PPF and then adding a high concentration of S1P (instead of exposing MKs to an S1P gradient), we found a significant reduction in the number of MKs displaying PP extensions (Fig. 5 A). Using differential interference contrast (DIC) microscopy of cultured MKs, we observed that exposure of MKs to a high, homogenous concentration of S1P results in almost immediate shedding of platelet-like particles from PPs (Fig. S1 B andVideo 4). Within 1 h, plateletlike particles were shed from $26 \%$ of PPs in response to S1P but only from 3\% of PPs treated with vehicle (Fig. 5 B). To further quantify the effect of S1P on PP shedding in vitro, we determined the number of fragmentation events by flow cytometry (Fig. 5 C). S1P, but not vehicle, increased PP fragmentation at high S1P concentrations, mimicking S1P plasma levels but not at low concentrations prevailing in the BM interstitium (Fig. 5, C and D).

In vivo, blood flow-induced shear stress might facilitate the separation of intravascular cell fragments from MKs (Junt et al., 2007). We therefore evaluated whether S1P also plays a role for PP fragmentation under flow conditions. Cultured MKs exposed to the physiological shear stress of BM sinusoids (4 dynes $/ \mathrm{cm}^{2}$; Junt et al., 2007) in the absence of S1P (serum-free buffer) rarely shed PPs from their MK stems. In contrast, in the presence of $5 \mu \mathrm{M} \mathrm{S1P}$, PPs were rapidly released (Fig. 5, E and F; and Video 5), indicating that S1P is required for $\mathrm{PP}$ shedding under static as well as flow conditions. Loss of S1pr2 or S1pr4 did not affect S1P-induced PP shedding (Fig. 5 B, Fig. S1 B, and Video 6). However, lack of the megakaryocytic S1pr1 receptor completely abolished S1P-induced release of PPs (Fig. 5 B, Fig. S1 B, and Video 6). This indicates that S1pr1, but not S1pr2 or S1pr4, plays the predominant role for S1P-driven PP shedding. To further clarify the involved signaling pathway, we used pertussis toxin and NSC23766 to inhibit Gi and Rac GTPase activity, respectively. Both inhibitors blocked S1P-induced fragmentation of PPs (Fig. $5 \mathrm{~B}$ and Fig. S1 B). The observation that S1P activates Rac GTPase in MKs via S1pr1 (Fig. 4, E and F) together with the aforementioned findings suggests that S1P-induced PP fragmentation depends on S1pr1/Gi/Rac GTPase signaling.

\section{S1P controls PP shedding into blood via S1pr1 in vivo}

To address whether S1P-S1pr1 signaling is also essential for $\mathrm{PP}$ fragmentation in vivo, we examined PP shedding in live mice by MP-IVM. PP shedding from MKs was a frequent event in naive (nontransplanted) $\mathrm{S} 1 \mathrm{pr} 1^{+/+} \mathrm{CD} 41-\mathrm{YFP}^{\mathrm{ki} /+}$ transgenic mice (Junt et al., 2007) but also in S1 pr1 ${ }^{+/+}$CD41YFPki/+ BM chimaeras (Video 7). Most MKs shed PP fragments that consist of beaded platelet-like structures (Fig. 6, $A$ and B; and Video 7), which generate mature platelets by undergoing consecutive fragmentation steps (Behnke and Forer, 1998; Junt et al., 2007). More than $60 \%$ of the S1 pr1 $1^{+/+}$ MKs carrying intravascular PP processes showed fragmentation within $1 \mathrm{~h}$ (Fig. 6, A and B; andVideo 7). We did not find any defect in PP fragmentation in $\mathrm{S} 1 \mathrm{pr} 2^{-/-}$or S1pr4 $4^{-/-}$ mice (Fig. $6 \mathrm{~A}$ ), whereas this process was severely impaired in S1pr1 mutants. In S1pr1 ${ }^{-/-}$chimaeras, we barely observed intravascular PP processes because of the aberrant interstitial PPF reported above (Fig. 4, H and I). However, 70-100\% of the PP processes that had eventually made their way into BM

absence of $50 \mu \mathrm{M}$ NSC23766, a Rac GTPase inhibitor (4,000-7,000 MKs per experiment; three independent experiments with triplications). (H) Representative MP-IVM images of MKs with YFP+ or EGFP+ PPs. Green indicates MKs and PPs; red indicates sinusoids. Arrowheads indicate extravascular YFP+ or EGFP+ PPs; arrows indicate interstitial YFP+ or EGFP+ PPs. The inset shows magnification for the dotted box. The arrow in the inset indicates the connection between extravascular YFP+ PPs. Bars: (C) $2 \mu \mathrm{m}$; (H) $20 \mu \mathrm{m}$. (I) MKs displaying intrasinusoidal PPF in vivo presented as a percentage of all MKs carrying PPs (20-30 MKs per group; five independent experiments for WT; three independent experiments for other genotypes). (J) The lateral (x-y) speed of PP

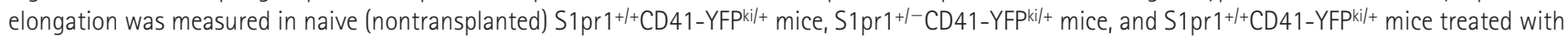
W146. Red lines indicate means. Data are pooled from three mice each group. All error bars indicate SEM. 
A

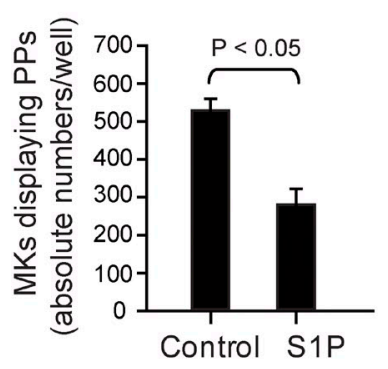

B

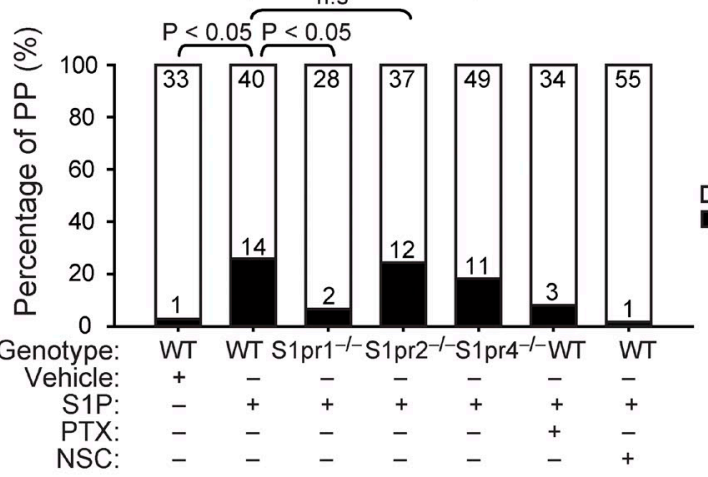

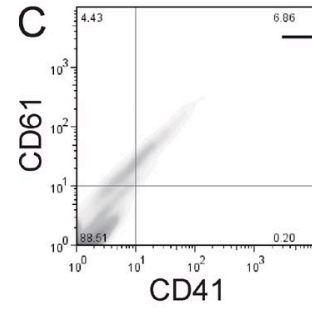
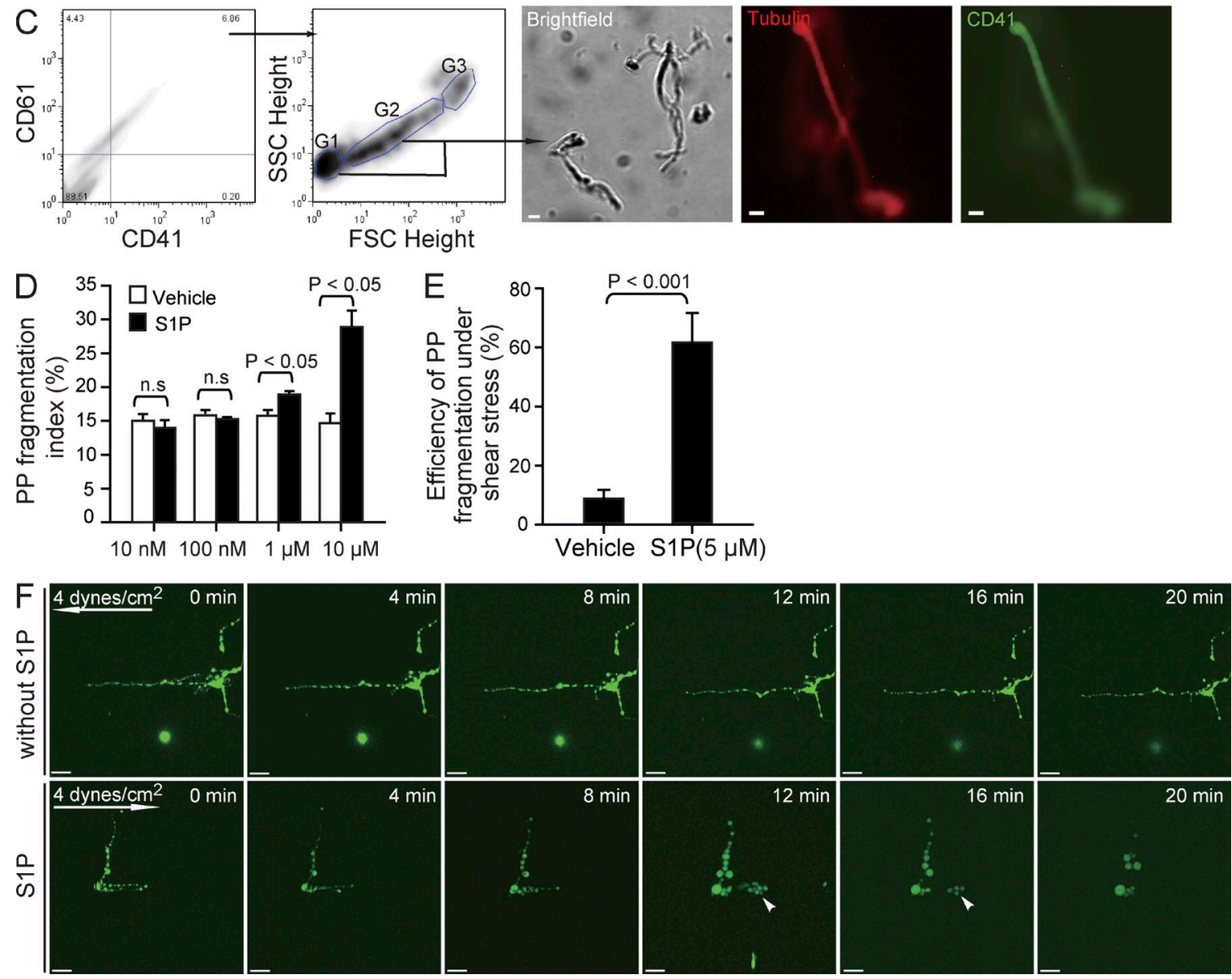

Figure 5. The effect of S1P on PP fragmentation in vitro. (A) The number of MKs displaying PPF in the absence or presence of 10 $\mu$ M S1P (230-590 MKs per experiment; three independent experiments with triplications). (B) The number of PPs with or without fragmentation observed by DIC microscopy in vitro over $1 \mathrm{~h}$ in the indicated groups. Data are pooled from 4-10 independent experiments for each group ( $n=30-60$ per group). (C) Representative dot plots show flow cytometric analyses of PP fragmentation. The first two panels show the gates for PPs. The CD41+CD61+ population was analyzed for the distribution of PPs according to FSC and SSC. MKs are G3; PPs with higher and lower FSC values are G2 and G1, respectively. The three representative microphotographs in the right show a representative brightfield image, as well as tubulin and CD41 stainings of fragments sorted using the gating strategy illustrated in the two plots. (D) Flow cytometric analyses of the PP fragmentation index in the presence or absence of various concentrations of S1P. The PP fragmentation index was calculated as described in Materials and methods. Data are representative of six independent experiments with triplication. (E) PP fragmentation by MKs exposed to shear stress. The efficiency of dynamic PP fragmentation was calculated as described in Materials and methods. Data are pooled from five independent experiments for each group. (F) Representative time-lapse video microscopy of PPs in the presence or absence of $5 \mu \mathrm{M}$ S1P under shear stress $\left(4 \mathrm{dynes} / \mathrm{cm}^{2}\right)$. Arrows indicate direction of flow; arrowheads indicate PP shedding events. All error bars indicate SEM. Bars: (C) $10 \mu \mathrm{m}$; (F) $20 \mu \mathrm{m}$. 

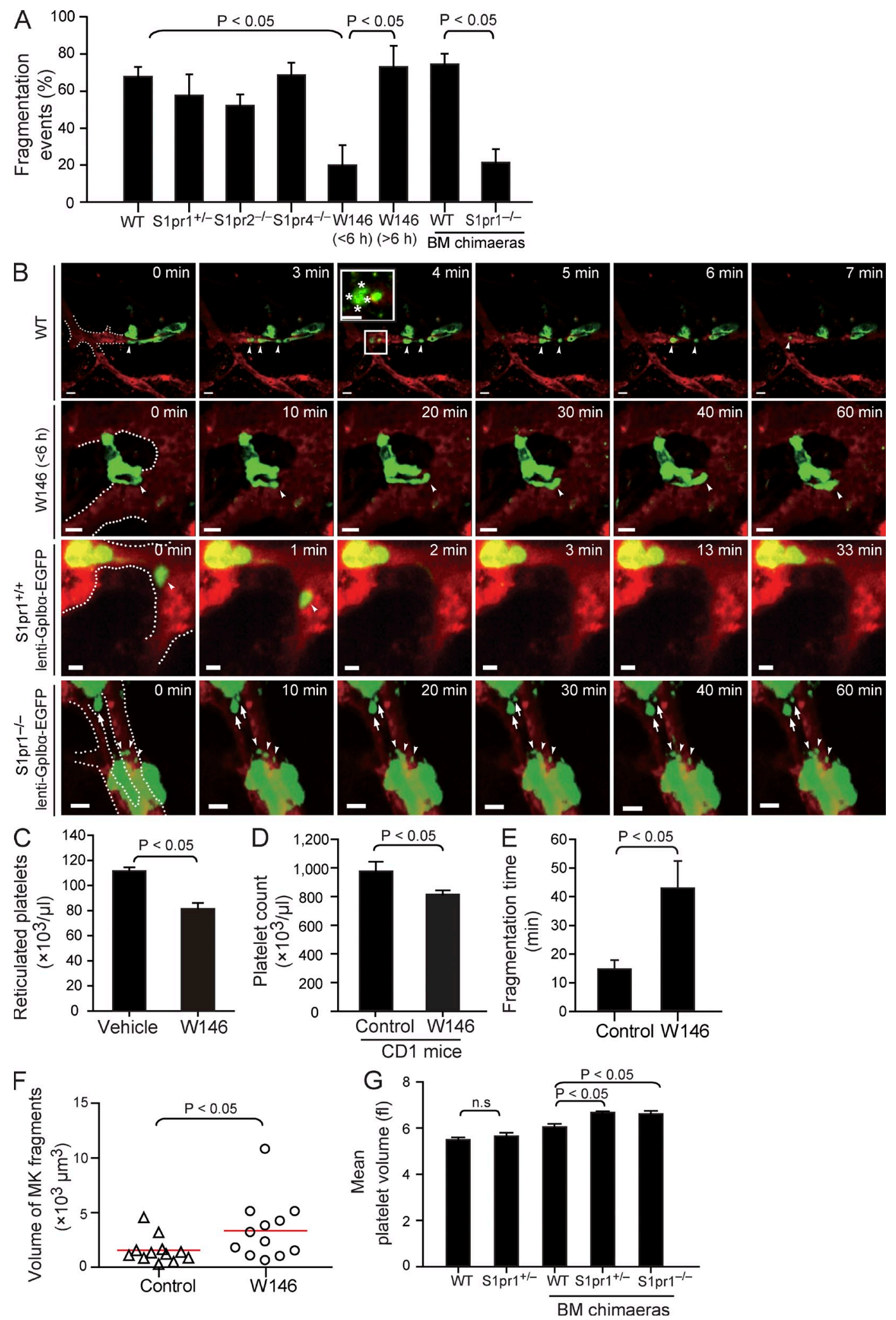

Figure 6. The effect of S1P on PP fragmentation in vivo. (A) Percentage of PP fragmentation events observed by MP-IVM over $1 \mathrm{~h}$ in the indicated groups. $n=13-33$ per group. Data are pooled from three to seven independent experiments. (B) Role of S1 pr 1 for PP shedding in vivo visualized by MP-IVM. Representative MP-IVM sequences show that WT MKs frequently shed PPs as shown in the first and the third rows. The inset shows a magnification of a shed PP particle. Asterisks show embedded platelet-like particles. Inhibition or loss of S1 pr 1 abolishes PP shedding (second and fourth rows). Arrowheads indicate intrasinusoidal PPs, and arrows show extrasinusoidal PPs in S1 pr1 $1^{-1-}$ chimaeras. The dashed lines highlight the sinusoids. Green or yellow 
sinusoids remained firmly attached to their MK stems; only in rare instances did MKs release PP fragments (Fig. 6, A and B; and Video 7). Together, these data indicate that S1pr1 is critical for both directional PPF and for proper intravascular PP fragmentation. Defective PP shedding is likely to explain the increase in size of S1 pr1 $1^{-/-}$MKs (Fig. 3 E). Interestingly, the frequency of intravascular PP shedding was only moderately reduced in CD41-YFPki/+ S1 pr1 ${ }^{+/-}$mice (Fig. $6 \mathrm{~A}$ ), suggesting that a single S1pr1 allele is sufficient to maintain intravascular PP shedding and that the mild thrombocytopenia observed in S1pr1 ${ }^{+/-}$mice is mostly caused by a defect in navigating PP processes into BM sinusoids (Fig. 4, H and I).

To examine whether S1pr1 regulates the dynamic process of PP shedding independently from its effects on PP invasion into BM sinusoids, we next tested the consequences of shortterm pharmacological inhibition of S1pr1. We treated naive (nontransplanted) S1 $\mathrm{pr}^{+/+}$CD41-YFPki/+ mice with a single dose of the selective S1pr1 antagonist W146 and visualized PP shedding immediately thereafter. In contrast to protracted inhibition or genetic ablation of S1pr1 (Fig. 4, H and I), this did not affect the overall number of MKs with intravascular PP protrusions. However, $<20 \%$ of MKs with established intravascular protrusions managed to release PP fragments into the blood stream within $6 \mathrm{~h}$ after administration of W146; the vast majority of the intrasinusoidal processes remained attached to their MK stems (Fig. 6,A and B; and Video 8). Repetitive treatment of mice with W146 for $24 \mathrm{~h}$ resulted in a significant reduction in circulating young reticulated platelets with an elevated RNA content (Fig. 6 C), consistent with a central role of the S1PS1pr1 pathway for PP fragmentation and release of platelets. Short-term treatment with W146 also reduced platelet counts in CD1 mice (Fig. 6 D), suggesting that S1pr1 controls thrombopoiesis across different strains of mice. W146 maintains an adequate in vivo receptor blockade for only 5-6 h (Sanna et al., 2006), and shedding reoccurred $6 \mathrm{~h}$ after a single dose of W146, suggesting that S1pr1 inhibition does not affect the viability of MKs (Fig. $6 \mathrm{~A}$ and Video 8). In rare instances, where PP shedding occurred in the presence of the S1pr1 inhibitor W146, the time required until an intravascular fragment dissociated from its MKs stem was significantly prolonged (Fig. 6 E). The failure to properly shed PPs resulted in the formation of abnormal, thick intravascular PP processes (Video 8). In line with this observation, the few PP fragments that were released despite the presence of W146 were significantly bigger compared with those in vehicle-treated control mice (Fig. 6 F), reminiscent of the large platelets observed in S1 pr1-null chimaeras (Fig. 6 G and Table S2).

\section{S1pr1 agonists enhance platelet production}

Modulation of S1P receptors by FTY720 (fingolimod) has become a promising strategy for the treatment of patients with multiple sclerosis (Kappos et al., 2006). Here, we show that treatment of mice with a single dose of FTY720 leads to a prompt and transient increase in circulating platelets (Fig. 7 A). When we used MP-IVM to examine MKs before and after treatment with a single dose of FTY720, we found that FTY720 accelerates the shedding of intravascular PP extensions into the blood stream. As a consequence, the number of MKs carrying intravascular PPs significantly decreased immediately after a single dose of FTY720 compared with vehicle (Fig. 7, B and C). This suggests that FTY720 represents an agonist for megakaryocytic S1pr1 receptors and has the potential to rapidly mobilize PPs into the blood, most likely by supporting fragmentation of intravascular PPs (Fig. 7 , B and C).Treatment with the S1pr1-specific agonist SEW2871 also caused an increase in circulating blood platelets (Fig. 7 D), further supporting that activation of S1P-S1pr1 receptor signaling enhances thrombopoiesis.

\section{DISCUSSION}

Our results assign a new role for S1P and its receptor S1 pr1 as master regulators of thrombopoiesis. In a dose-dependent and sequential manner, S1P controls two key steps in the cascade of thrombopoiesis by BM MKs: (1) the polarized development of PP extensions into the blood stream and (2) the subsequent shedding of PPs from their transendothelial stems. As a consequence, loss of S1pr1 is not compatible with normal thrombopoiesis. Collectively, our findings uncover the molecular pathway that enables the final steps of thrombopoiesis.

\section{S1P navigates PP extensions into BM sinusoids and initiates platelet release}

Mature MKs form intravascular PP extensions that grow from the MK cell body at a mean speed of $10 \mu \mathrm{m} / \mathrm{min}$ under shear conditions in vivo (Fig. $4 \mathrm{~J}$ ), with the DMS functioning as the membrane reservoir for PP elongation (Schulze et al., 2006). During elongation, PPs are equipped with specific proteins associated with platelets, including von Willebrand factor $(\mathrm{vWF})$ and fibrinogen receptors. Microtubules, assembled from $\alpha / \beta$-tubulin dimers, are the primary structural component of the engine that drives the elongation of PPs. Correspondingly, PPs fail to form when cultured MKs are exposed to agents that inhibit microtubule assembly (Italiano et al., 1999) or sliding (Patel et al., 2005b), and mice lacking $\beta 1$-tubulin,

indicates MKs and PPs; red indicates sinusoids. Bars, $20 \mu \mathrm{m}$. (C) Circulating reticulated (young) platelets in mice treated with W146, an S1pr1-specific antagonist, or vehicle as assessed by flow cytometry. $n=3$ mice each group. (D) Circulating platelet counts in CD1 mice treated with W146 or vehicle. $n=4$ mice each group. (E) The time point of fragmentation detected by MP-IVM in mice treated with W146 (<6 h) or vehicle. Data are pooled from three independent experiments each group. (F) Volumes of PP fragments in mice treated with W146 (within 6 h) or vehicle. Red lines indicate means. Data are pooled from three independent experiments each group. (G) Mean platelet volume in the indicated genotypes. $n=8$ for WT; $n=6$ for S1pr $1^{+1-} ; n=15$ for WT BM chimaeras; $n=9$ for S1pr1 ${ }^{+1-}$ BM chimaeras; $n=14$ for S1 pr1 $1^{-1-}$ BM chimaeras. All values are mean and SEM. 
A
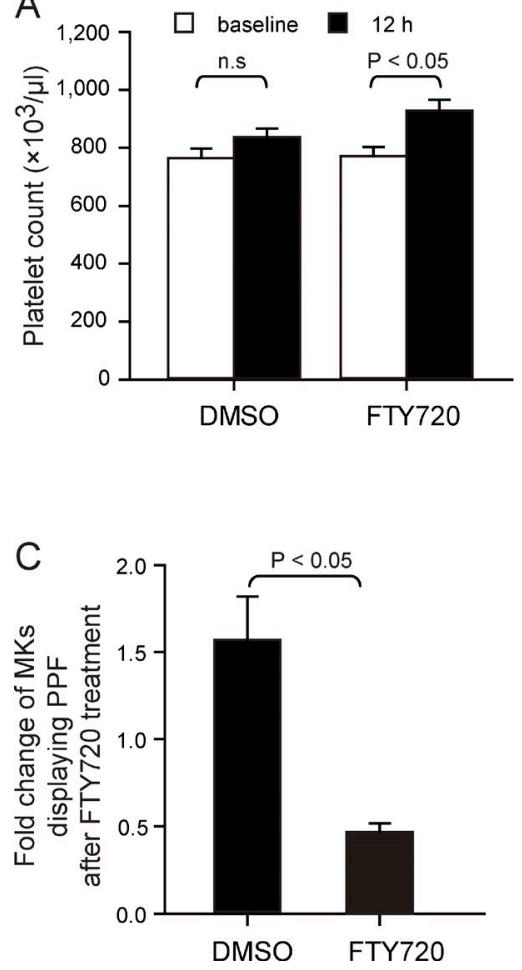

B
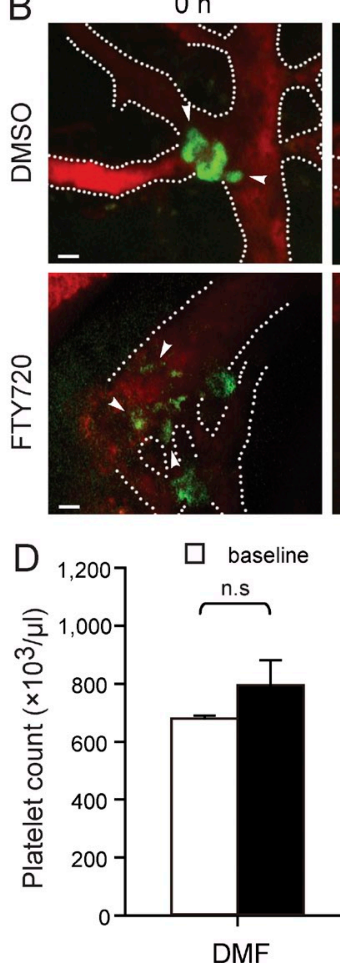

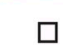

$\square$ baseline
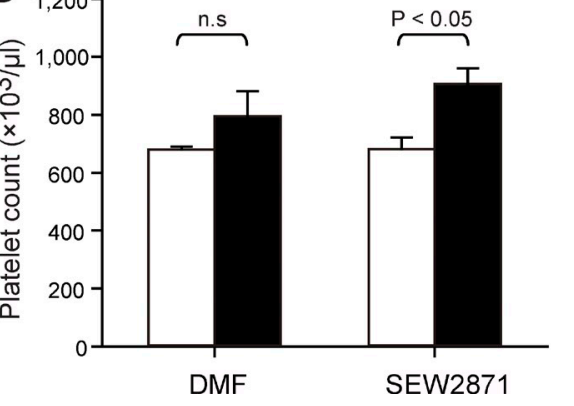

Figure 7. The S1P analogue FTY720 and SEW2871 trigger rapid release of platelets. (A) Mice were treated with a single dose of FTY720 (3 mg/kg i.p.) or DMSO (vehicle). Open bars indicate baseline of platelet counts assessed before drug administration; closed bars indicate platelet counts measured $12 \mathrm{~h}$ after drug administration. $n=9$ mice each group. (B) Representative MP-IVM images of MKs with YFP+ PPs from CD41-YFPkil+ mice before and $8 \mathrm{~h}$ after a single injection of FTY720 (3 mg/kg i.p.) or DMSO (vehicle). Green indicates MKs and PPs; red indicates sinusoids. Arrowheads indicate intravascular PPs. Dashed lines indicate BM sinusoidal vessels. Bars, $20 \mu \mathrm{m}$. (C) Fold change of MKs displaying PPF $8 \mathrm{~h}$ after a single injection of FTY720 (3 mg/kg i.p.) or DMSO (vehicle) compared with numbers recorded before treatment. $n=120-140$ MKs per group. Data are pooled from three independent experiments. (D) Mice were treated with a single dose of SEW2871 (20 mg/kg i.p.) or dimethyl formamide (DMF; vehicle). Open bars indicate baseline of platelet counts assessed before drug administration; closed bars indicate platelet counts measured $12 \mathrm{~h}$ after drug administration. $n=3$ mice each group. All error bars indicate SEM. the most abundant $\beta$-tubulin in platelets, develop thrombocytopenia (Schwer et al., 2001).

Cultured MKs form PPs that elongate into random directions (Italiano et al., 1999; Dunois-Lardé et al., 2009). In contrast, we show here by MP-IVM that PPF occurs in a highly polarized fashion in the BM in vivo. This suggests that PPs integrate a previously unknown guidance signal, which navigates them into the intravascular compartment and avoids the interstitial BM compartment. Our study has uncovered this guidance signal and shows that a transendothelial S1P gradient in the BM controls the directionality of PPF and elongation. We observed that the interstitial S1P concentrations in the $\mathrm{BM}$ are low, whereas the S1P blood concentration is orders of magnitude higher. Residing at the vascular interface, mature MKs are located in a particularly strategic position for integrating the guidance cues provided by the transendothelial S1P gradient. Equipped with S1pr1, they sense the steep vascular S1P gradient and extend dynamic PP protrusions into microvessels along increasing concentrations of this lipid mediator. A similar S1P gradient also exists in the lymph node between lymph node interstitium and the lymph fluid, where it drives the migration of $\mathrm{T}$ cells into efferent lymph vessels (Matloubian et al., 2004). Recent observations showing that the S1P pathway controls the egress of lymphocytes from the $\mathrm{BM}$ into the blood stream emphasize the biological relevance of the transendothelial S1P gradient for BM homeostasis (Allende et al., 2010).

Once MKs have successfully extended their PPs into the blood, they release fragments from the tips of their intravascular projections (Video 7; Junt et al., 2007). These new PP fragments break down further in the circulation giving rise to mature platelets of $2-3-\mu \mathrm{m}$ diameter within the circulation (Stenberg and Levin, 1989). Blood shear stress contributes to the shedding of PPs (Junt et al., 2007; Dunois-Lardé et al., 2009; Thon et al., 2010); however, whether additional signals are required for efficient PP shedding was completely unknown. In this study, we show that hydrodynamic forces alone are not sufficient to allow the release of new platelets from MK PP extensions. Instead, we found that high concentrations of the bioactive lipid S1P prevailing in the sinusoidal blood, but not in the BM interstitium, are mandatory for the release of new platelets from MKs.

From a teleological point of view, the S1P-dependent sequential guidance of thrombopoiesis comprising (a) directional PPF along a transendothelial S1P gradient and (b) subsequent S1P-dependent intravascular PP shedding leads to the introduction of naive platelets into the circulating blood and prevents aberrant platelet production within the BM interstitium. S1P guidance of intravascular PPF, elongation, and shedding therefore provides grounds for efficient thrombopoiesis, which seems instrumental given the relative paucity of MKs.

\section{S1P controls thrombopoiesis via megakaryocytic S1pr1 receptors}

Our study shows that MKs robustly express three different $\mathrm{S} 1 \mathrm{P}$ receptors, S1 pr1, S1 pr2, and S1pr4. Loss of S1pr1 on hematopoietic cells and also conditional deficiency of S1pr1 in MKs were associated with severe thrombocytopenia. Moreover, gain of S1pr1 function in S1pr1 $1^{-/}$MKs rescued their 
defect in platelet production. These results clearly demonstrate that S1pr1 expressed by the MK lineage intrinsically controls platelet homeostasis. It has been shown previously that signaling via S1pr1 activates Rac GTPases in multiple hematopoietic lineages, including $\mathrm{T}$ cells (Matsuyuki et al., 2006; Gérard et al., 2009). Consistent with this, we observed here that Rac activation is triggered in MKs by S1pr1 agonists. Rac GTPases are known to regulate actin dynamics and induce the formation of membrane extensions (Aspenström et al., 2004). In MKs, the turnover of actin filaments is known to control platelet formation (Bender et al., 2010). Correspondingly, we found here that Rac GTPase activation leading to cytoskeletal reorganization is indispensable for S1P-S1pr1-driven PPF and fragmentation. Although we observed that simultaneous pharmacological inhibition of all Rac GTPases by NSC23766 virtually abolishes S1P-driven thrombopoiesis in vitro, loss of Rac1 alone does not lead to thrombocytopenia in vivo (McCarty et al., 2005), suggesting that other Rac GTPase family members, including Rac2, Rac3, and RhoG, may have redundant functions in thrombopoiesis and its control by S1P. In addition, we cannot rule out that other small Rho GTPases, including cdc42 and RhoA (Pleines et al., 2010; Pleines et al., 2012), also contribute to S1P-driven platelet generation by MKs.

Unlike the loss of $S 1 p r 1$, genetic disruption of $S 1 p r 2$ or S1pr4 on hematopoietic cells was not associated with thrombocytopenia. Moreover, loss of S1pr2 or S1pr4 did not result in any gross defect of $\mathrm{MK}$ development, directional PPF or PP fragmentation in vitro or in vivo. In line with this finding, one recent study demonstrated that S1pr4-deficient mice have normal numbers of BM MKs and normal platelet counts under physiological conditions (Golfier et al., 2010). Despite its irrelevance for maintenance of physiological blood platelet counts, S1pr4 was reported to play a subtle role in the terminal differentiation of MKs and PPF (Golfier et al., 2010), an effect which we did not observe here in several sets of in vitro and in vivo assays, most likely because of the different experimental conditions. Collectively, our present study and the previous study (Golfier et al., 2010) suggest that S1pr1, but not S1pr2 or S1pr4, plays a primary role in the control of thrombopoiesis under physiological conditions.

In contrast to other cells within the BM compartment, including osteoclast precursors (Ishii et al., 2009) and lymphocytes (Allende et al., 2010), MKs clearly do not require S1pr1 signaling for migration and positioning. This corroborates previous studies showing that these processes are predominantly orchestrated by fibroblast growth factor-4 (FGF-4) and SDF-1 (Majka et al., 2000; Avecilla et al., 2004). Likewise, loss of S1pr1 does not affect proliferation and maturation of megakaryocytic progenitor cells into platelet-producing MKs. This is consistent with the concept that megakaryopoiesis is regulated predominantly by TPO (Kaushansky, 2005b).

\section{S1P receptor agonist increases blood platelet counts}

Recently, modulation of S1P receptor signaling by FTY720 (fingolimod) has emerged as a promising immunosuppressive strategy and is currently being used in patients with relapsing multiple sclerosis (Kappos et al., 2006). After administration, FTY720 is metabolized to phosphorylated FTY720 (FTY720P), an agonist for four of the five S1P receptors including S1pr1. FTY720 limits effector lymphocyte egress from lymph nodes (Matloubian et al., 2004), contributing to its immunosuppressive actions. However, FTY720 has not been examined for its potential effects on megakaryo- and thrombopoiesis. In this study, we show that treatment of mice with a single dose of FTY720 leads to shedding of intravascular PP extensions into the blood stream, paralleled by a prompt, but transient increase in circulating platelets. This suggests that FTY720 acts as an agonist on megakaryocytic S1pr1 receptors and has the potential to rapidly mobilize PPs into the blood, most likely by supporting fragmentation of intravascular PPs. Whereas lymphocyte S1pr1 engagement by phosphorylated FTY720 within secondary lymphoid organs triggers down-modulation of the receptor, resulting in functional antagonism of the S1pr1 pathway, an agonistic effect of FTY720 similar to the one observed here for MK has recently been reported to promote the recirculation of BM osteoclast precursor monocytes from the bone surface (Ishii et al., 2009). This indicates that FTY720 predominantly exerts agonist effects in cells of the myeloid lineage. Because activation of S1P-S1pr1 receptor signaling enhances thrombopoiesis in mice, future studies will have to evaluate potential clinical implications of S1pr1 agonists, in particular in the treatment of thrombocytopenia.

Collectively, the present study reveals that $\mathrm{S} 1 \mathrm{P}$, a signaling lipid circulating in the blood, regulates dynamic intravascular PP elaboration and PP shedding without affecting MK maturation and positioning. Tonic S1P-S1pr1 signaling is critical for normal thrombopoiesis in mice. Although the exact role of S1P-S1pr1 signaling for human thrombopoiesis still needs to be defined, our findings could have clinical implications and provide new approaches to treat thrombocytopenia.

\section{MATERIALS AND METHODS}

Mice. C57BL6/J (CD45.2), B6.SJL-Ptprc ${ }^{a}$ Pep $3^{b} /$ BoyCrl (CD45.1), and CD1 mice were purchased from Charles River. $\beta$-Actin-EGFP mice were provided by A. Wagers (Harvard Medical School, Boson, MA). S1pr1 $1^{+/-}$and S1 pr2 ${ }^{-/-}$mice were generated as described previously (Liu et al., 2000; Kono et al., 2004). S1 pr4 ${ }^{-/-}$mice were provided by D. Guerini (Novartis Institutes for BioMedical Research, Basel, Switzerland). CD41-YFPki/+ mice were generated as described previously (Zhang et al., 2007). Pf4-cre and ROSA26flox-stop-flox-EYFP mice were obtained from the Jackson Laboratory and crossed to get Pf4-EYFP transgenic mice, in which EYFP is driven by the MK-specific Pf4 promoter. FL chimaeras and BM chimaeras were generated as described previously (Massberg et al., 2007). Cytometric analysis showed that $>95 \%$ of the blood cells were derived from donors in all the BM chimaeras. S1 pr1 $1^{\mathrm{fl} / \mathrm{fl}}$ mice were obtained from R.L. Proia. Age- and gendermatched mice in a C57BL/6 background were used in all experiments. All experimental procedures performed on animals met the requirements of the German legislation on the protection of animals.

Blood cells and serum TPO measurements. We measured blood cell counts in the mice before and $12 \mathrm{~h}$ after a single injection of FTY720 (3 mg/kg i.p.; Cayman) or DMSO (Sigma-Aldrich) as vehicle. Platelet counts were assessed in the mice before and $12 \mathrm{~h}$ after a single injection of SEW2871 (20 mg/kg i.p.; Cayman) or dimethyl formamide (Sigma-Aldrich). Serum TPO was measured using the Quantikine murine TPO Immunoassay kit 
(R\&D Systems). To block S1pr1 in vivo, we treated C57BL6/J or CD1 mice with W146 (3 mg/kg body weight i.p.) or vehicle every $6 \mathrm{~h}$ and measured platelet counts in these mice $24 \mathrm{~h}$ later.

Immunostaining. Anti-mouse S1pr1 (Zytomed), anti-human S1PR1 (ABR), anti-S1pr2 (Santa Cruz Biotechnology, Inc.), anti-S1pr4 (Santa Cruz Biotechnology, Inc.), antitubulin (Cell Signaling Technology), anti-mouse CD41 (BD), and anti-CD42c (Emfret Analytics) antibodies were used for immunostaining. The samples were examined using Leica microscopy equipped with $40 \times$ objective lens (NA $=0.7$ ) or $20 \times$ objective lens $(\mathrm{NA}=0.5$ ) and commercial charge-coupled device camera (AxioCam; Carl Zeiss). Images were acquired by AxioVision software (Carl Zeiss). For quantification of MK number and size in BM, we counted the total number of MKs in five randomly selected $20 \times$ microscopic fields, and the area of MK cell body was analyzed by ImageJ software (National Institutes of Health).

S1P level measurement. Levels of S1P were determined by highperformance liquid chromatography with mass detection as described previously (Berdyshev et al., 2005).

Transmission electron microscopy. BM samples were fixed in $2.5 \%$ glutaraldehyde, embedded in epon, and analyzed using an electron microscope (EM 902; Carl Zeiss).

Quantitative RT-PCR. Quantitative real-time PCR was performed using the MaximaTM SYBR Green PCR Master mix (Thermo Fisher Scientific) and an ABI PRISM 7700 Sequence Detection System (Applied Biosystems) according to the manufacturers' instructions. The data were normalized to the mRNA level of glyceraldehyde 3-phosphate dehydrogenase (Gapdh or GAPDH). Primers are listed in Table S1.

MK culture. Mouse embryonic day (E) 12.5-14.5 FL cells were cultivated in TPO-containing medium for differentiation into MKs (Lecine et al., 1998). In brief, mouse FLs were isolated from E12.5-14.5 embryos and kept in DMEM (Invitrogen) supplemented with 10\% charcoal-treated FBS (PAN) in the presence of hTPO (ImmunoTools) in a humidified 5\% $\mathrm{CO}_{2} / 95 \%$ air incubator at $37^{\circ} \mathrm{C}$. For isolation of immature MKs, E12.5$14.5 \mathrm{FL}$ cells were cultured in medium for $12 \mathrm{~h}$ and then incubated with FITC-CD41 (BD) and PE-CD34 (BioLegend) antibodies. Immature MKs were identified as a CD41/CD34 double-positive population and enriched by using a FACSAria cell sorter (BD). Mature MKs were enriched by BSA gradient, as described previously (Pang et al., 2006). In brief, the BSA step gradient was prepared by placing PBS containing $1.5 \%$ BSA on top of PBS with $3 \%$ BSA (PAA). Cells were loaded on top of the gradient, and MKs were settled to the bottom within $30 \mathrm{~min}$ at $1 \times$ gravity at room temperature. Mature MKs formed a pellet at the bottom of the tube. We distinguished and scored PPs according to the criteria mentioned previously (Chen et al., 2007). To inhibit the activity of Rac GTPases, we cultured MKs in the presence of $50 \mu \mathrm{M}$ NSC23766 (Tocris Bioscience) for $3 \mathrm{~d}$ and then scored MKs carrying PPs. To establish a stable gradient during the slow process of PPF, we applied chemotaxis $\mu$-slides (ibidi), which consist of two large reservoirs with different chemoattractant concentrations and a thin $(1 \mathrm{~mm})$ slit between these two reservoirs. A linear and stable chemoattractant gradient is established in the slit by diffusion (Zengel et al., 2011). MKs were resuspended in serum-free medium in the presence or absence of $1 \mu \mathrm{M}$ VPC23019 (Avanti Polar Lipids, Inc.) and loaded onto chemotaxis $\mu$-slides coated with poly-L-lysine between two large reservoirs. $10 \mu \mathrm{M}$ S1P or vehicle was applied into one of the reservoirs of the $\mu$-slides. MKs were cultivated for $10 \mathrm{~h}$ at $37^{\circ} \mathrm{C}$, and polarization of PPF was evaluated based on the images captured using an Axiovert microscope (Carl Zeiss) equipped with $20 \times$ objective lens (Nikon) and AxioVision software.

CFU-MK assay. We seeded 10,000 isolated BM or FL mononuclear cells into Megacult-C medium (STEMCELL Technologies) and scored CFUMKs on day 5 according to the manufacturer's instructions.
Lentiviral infection of FL or BM cells. The different lentiviral constructs were derived from the pTRIP $\Delta \mathrm{U} 3 \mathrm{EF} 1 \alpha$-EGFP vector (a gift from P. Charneau, Institut Pasteur, Paris, France). The EF1 $\alpha$ promoter was replaced by a $541-b p$ core promoter fragment ( -254 to 287 ) of the murine GPIb $\alpha$ promoter to obtain the vector pTRIP $\Delta \mathrm{U} 3 \mathrm{mGPIb} \alpha-\mathrm{EGFP}$. The GFP open reading frame was removed, and the coding region for murine S1pr1 was inserted to obtain the vector pTRIP $\Delta \mathrm{U} 3 \mathrm{mGIb} \alpha-\mathrm{mS} 1 \mathrm{pr} 1$. A subcloned DNA fragment containing a 921-bp fragment of the human GPII promoter $(-889$ to 32), and the Cre coding sequence was directly cloned into the vector pTRIP $\Delta \mathrm{U} 3 \mathrm{EF} 1 \alpha$-EGFP to obtain the vector pTRIP $\Delta \mathrm{U} 3$ hGPIIb-Cre.

$\mathrm{BM}$ or FL cells were incubated with lentiviral particles in the presence of $8 \mu \mathrm{g} / \mathrm{ml}$ polybrene (Sigma-Aldrich) at $37^{\circ} \mathrm{C}$ for $12 \mathrm{~h}$ in serum-free medium supplemented with $1 \%$ BSA, $100 \mathrm{ng} / \mathrm{ml}$ Flt3-ligand, $100 \mathrm{ng} / \mathrm{ml} \mathrm{stem}$ cell factor (Sigma-Aldrich), $20 \mathrm{ng} / \mathrm{ml}$ TPO (ImmunoTools). After transduction with lentiviral vectors, BM and FL cells were injected into irradiated mice (two doses of $6.5 \mathrm{~Gy}$ ). For reexpression of S1pr1 in S1 pr1 ${ }^{-/-} \mathrm{MKs}$, $\mathrm{S} 1 \mathrm{pr} 1^{-/-} \mathrm{FL}$ cells were transduced with lenti-GPIb $\alpha-\mathrm{S} 1 \mathrm{pr} 1$ viral vectors to express S1pr1 under the control of MK promoter GPIb $\alpha .10^{5} \mathrm{~S} 1 \mathrm{pr}^{-/-} \mathrm{FL}$ cells transduced with lenti-GPIb $\alpha-S 1$ pr1 or empty lentiviral vector and $2 \times$ $10^{6} \mathrm{BM}$ cells from $\beta$-actin-EGFP mice were coinjected into irradiated CD45.1 mice. The percentage of EGFP-positive platelets was determined by flow cytometry. Because the donor chimaerism in peripheral whole blood of all generated chimaeras was $>99 \%$ and $100 \%$ of platelets from $\beta$-actin-EGFP mice were EGFP positive, almost all EGFP-negative platelets were from $\mathrm{S} 1 \mathrm{pr}^{-/-}$FL donor cells. For conditional deletion of S1pr1 in MKs, S1 pr1 $1^{\mathrm{t} / \mathrm{fl}}$ BM cells were transduced with lenti-GPIIb-cre viral vectors to express Cre recombinase under the control of MK promoter GPIIb and transferred into irradiated mice. S1pr1 floxed allele was excised in MKs in S1 pr $1^{\mathrm{A} / \mathrm{H}}$ BM chimaeras.

Flow cytometry. We analyzed the mean platelet sizes as described previously (Gramaglia et al., 2005). We performed platelet life span assays as described previously (Robinson et al., 2000). We used Thiazole orange (Molecular Probes) to stain residual RNA in juvenile, reticulated platelets and detected them by flow cytometry as described previously (Matic et al., 1998). For analysis of PP fragmentation, FL-derived MK cultures were treated with various concentrations of $\mathrm{S} 1 \mathrm{P}$ or vehicle for $4 \mathrm{~h}$ and then stained with CD41-FITC and CD61-PE (BD) antibodies. The PP fragmentation index is determined by the percentage of G1 in the PP population (G1 + G2; Fig. 5 C). We collected the PP fraction according to the gate in Fig. 5 C using a FACSAria cell sorter and then observed the sorted PPs using brightfield microscopy (Fig. 5 C).

Western blot analyses. Y10/L8057 mouse megakaryocytic cells were cultured in IMDM supplemented with 10\% FCS and $25 \mathrm{ng} / \mathrm{ml}$ TPO for $1 \mathrm{~d}$ and then starved overnight on $100-\mathrm{mm}$ dishes coated with $0.5 \%$ fatty acid-free BSA (Sigma-Aldrich). The starved Y10/L8057 cells were simulated with $10 \mu \mathrm{M}$ S1P or vehicle for $2 \mathrm{~min}$ or $1 \mu \mathrm{M}$ SEW2871 or vehicle for $5 \mathrm{~min}$. RacGTP activities were measured using Rac assay kit (Cell Biolabs). Platelet lysates were subjected to SDS-PAGE and then immunoblotted with antibodies recognizing murine S1pr1 (Imgenex) or $\beta$-actin (Abcam) as loading controls.

Multiphoton intravital imaging of the BM. We prepared the mouse calvarian BM as described previously (Junt et al., 2007). We used a BioTech TriMScope system (LaVision BioTec) and Ti: Sa laser (MaiTai) to capture images through a $20 \times$ water immersion objective lens $(\mathrm{NA}=0.95$; Olympus). Images were acquired with ImSpectorPro (LaVision BioTec). For three-dimensional (3D) acquisition, the stacks were acquired at a $920-\mathrm{nm}$ wavelength at vertical spacing of $2-3 \mu \mathrm{m}$ to cover an axial depth of 30-100 $\mu \mathrm{m}$ (for YFP or EGFP). Subsequently, the same stacks were acquired at a wavelength of $800 \mathrm{~nm}$ (for TRITC-dextran). The distances between MKs and vasculatures were measured in the reconstructed 3D structure using Volocity software (PerkinElmer). If MKs were outside the vessels, the closest distance from MKs to vessels was measured and represented as negative values. If MKs were in direct physical contact with the vessels, the distance was regarded as 
zero. For analysis of PP shedding, four-dimensional acquisitions were performed at $920 \mathrm{~nm}$ by capturing 3D image stacks at an interval of $60 \mathrm{~s}$ for $60 \mathrm{~min}$. Videos were generated as maximum intensity projections representing a "top" $(\mathrm{x}-\mathrm{y})$ view of the volume using Volocity. The centroid positions ( $x-y)$ of MKs or PP tips from a series of top-view $(x-y)$ images were measured using ImageJ, and instantaneous lateral $(x-y)$ velocity, a measure of cell motility was determined by dividing the change in cell displacement between each frame by the time interval between frames and was quantified by the Chemotaxis and Migration Tool plugin (ibidi). All mice were treated with $8 \mu \mathrm{g} / \mathrm{kg} / \mathrm{d}$ mTPO (ImmunoTools) for $3 \mathrm{~d}$ before imaging as described previously (Junt et al., 2007). W146 (Avanti Polar Lipids, Inc.) or vehicle was injected (i.p.) $3 \mathrm{mg} / \mathrm{kg}$ body weight every $8 \mathrm{~h}$ for $24 \mathrm{~h}$ before imaging. To evaluate PP shedding, the mice were injected (i.p.) with W146 ( $3 \mathrm{mg} / \mathrm{kg}$ body weight) or vehicle and immediately visualized using MPIVM. For FTY720 experiments, the same MKs were visualized in mice before and $8 \mathrm{~h}$ after a single injection of FTY720 (3 mg/ $\mathrm{kg}$ i.p.) or DMSO using MP-IVM.

PP shedding under shear stress. MKs from $\beta$-actin-EGFP mice were seeded in $\mu$-slides VI coated with $100 \mu \mathrm{g} / \mathrm{ml}$ human fibrinogen (SigmaAldrich). The slides were then connected to a pump system (ibidi). A laminar shear stress of 4 dynes $/ \mathrm{cm}^{2}$ was applied to the cells in the presence of $5 \mu \mathrm{M}$ $\mathrm{S} 1 \mathrm{P}$ or vehicle. Image stacks were acquired at $2 \mu \mathrm{m}$ in $\mathrm{z}$ to cover a $20-\mu \mathrm{m}$ vertical distance at 60 -s intervals for $20 \mathrm{~min}$. The efficiency of PP fragmentation was determined by $\left(\mathrm{L}_{0 \text { min }}-\mathrm{L}_{20 \min }\right) / \mathrm{L}_{0 \min } \times 100 \% . \mathrm{L}_{0 \min }$ and $\mathrm{L}_{20 \text { min }}$ represent the length of PPs at $0 \mathrm{~min}$ and $20 \mathrm{~min}$, respectively.

Live cell imaging. Mature MKs were starved in serum-free medium in custom-made Petri dishes coated with $100 \mu \mathrm{g} / \mathrm{ml}$ of human fibrinogen (Sigma-Aldrich) for $4 \mathrm{~h}$ before incubation with S1P or vehicle. We treated MKs with $10 \mu \mathrm{M}$ S1P together with $25 \mu \mathrm{M}$ NSC23766 (Tocris Bioscience) to inhibit Rac GTPases. We incubated MKs with $500 \mathrm{ng} / \mathrm{ml}$ pertussis toxin (Sigma-Aldrich) to inhibit Gi signaling $1 \mathrm{~h}$ before the addition of $10 \mu \mathrm{M} \mathrm{S1P}$. Live cell imaging was performed as described previously (Lämmermann et al., 2008). In brief, MKs were kept on a heated micro-incubator to keep the temperature at $37^{\circ} \mathrm{C}$ and monitored using a DIC microscope system (Carl Zeiss), equipped with a $40 \times$ oil objective lens with $\mathrm{NA}=0.7$ (Carl Zeiss).

Statistics. We used two-tailed type 2 Student's $t$ test and KolmogorovSmirnov test to calculate $\mathrm{p}$-values. We considered p-values of $<0.05$ as statistically significant.

Online supplemental material. Fig. S1 shows intravascular PPF and DIC microscopy of PP fragmentation. Videos 1 and 2 show intravital visualization of PPF and shedding in WT or S1pr1 mutants. Video 3 shows intravital visualization of PPs in WT mice treated with S1pr1 inhibitor, W146, or its vehicle. Videos 4 and 5 show the effect of S1P on PP shedding in vitro. Video 6 shows the effect of S1P on PP shedding in S1pr2 ${ }^{-/-}$or S1pr4 ${ }^{-/}$MKs or S1 $\mathrm{pr}^{-1-}$ MKs. Videos 7 and 8 show intravital visualization of PP shedding in WT or S1pr1 mutants or WT mice treated with W146. Table S1 shows the sequences of primers used for qRT-PCR. Table S2 shows the blood cell counts in BM chimaeras. Table S3 shows the blood cell counts in nontransplanted mice. Online supplemental material is available at http://www.jem .org/cgi/content/full/jem.20121090/DC1.

We thank Dr. Timothy Hla (Cornell University, Ithaca, NY) for helpful discussions and suggestions.

This study was supported by the Deutsche Forschungsgemeinschaft and by the Intramural Research Program of the National Institutes of Health, National Institute of Diabetes and Digestive and Kidney Diseases.

A. Billich and T. Junt are employees of Novartis. The authors have no further conflicts of interest.

Author contributions: L. Zhang and S. Massberg came up with the conception and study design and wrote the manuscript; L. Zhang, M. Orban, M. Lorenz, V. Barocke, D. Braun, N. Urtz, C. Schulz, M.-L. von Brühl, A. Tirniceriu, F. Gaertner,
S.-S. Bolz, and A. Billich performed MP-IVM, generated chimaeras, generated lentiviral constructs, performed in vitro MK assays, generated FL-derived MKs, and performed S1P measurements; R.L. Proia and T. Graf generated and provided mutant mice and helped with data interpretation; M. Prinz and A. Müller performed electron microscopy; E. Montanez and M. Sixt performed in vitro shedding assay and examined S1 pr1 downstream signaling; L. von Baumbarten, R. Fässler, M. Sixt, U.H. von Andrian, and T. Junt helped with MP-IVM, data interpretation, and discussion.

Submitted: 22 May 2012

Accepted: 5 October 2012

\section{REFERENCES}

Allende, M.L., T. Yamashita, and R.L. Proia. 2003. G-protein-coupled receptor S1P1 acts within endothelial cells to regulate vascular maturation. Blood. 102:3665-3667. http://dx.doi.org/10.1182/blood-2003-02-0460

Allende, M.L., G. Tuymetova, B.G. Lee, E. Bonifacino, Y.P. Wu, and R.L. Proia. 2010. S1P1 receptor directs the release of immature B cells from bone marrow into blood.J. Exp.Med.207:1113-1124.http://dx.doi.org/ 10.1084/jem.20092210

Aspenström, P., A. Fransson, and J. Saras. 2004. Rho GTPases have diverse effects on the organization of the actin filament system. Biochem.J. 377:327-337. http://dx.doi.org/10.1042/BJ20031041

Avecilla, S.T., K. Hattori, B. Heissig, R. Tejada, F. Liao, K. Shido, D.K. Jin, S. Dias, F. Zhang, T.E. Hartman, et al. 2004. Chemokine-mediated interaction of hematopoietic progenitors with the bone marrow vascular niche is required for thrombopoiesis. Nat. Med. 10:64-71. http:// dx.doi.org/10.1038/nm973

Behnke, O., and A. Forer. 1998. From megakaryocytes to platelets: platelet morphogenesis takes place in the bloodstream. Eur. J. Haematol. Suppl. 61:3-23.

Bender, M., A. Eckly, J.H. Hartwig, M. Elvers, I. Pleines, S. Gupta, G. Krohne, E. Jeanclos, A. Gohla, C. Gurniak, et al. 2010. ADF/n-cofilindependent actin turnover determines platelet formation and sizing. Blood. 116:1767-1775. http://dx.doi.org/10.1182/blood-2010-03-274340

Berdyshev, E.V., I.A. Gorshkova, J.G. Garcia, V. Natarajan, and W.C. Hubbard. 2005. Quantitative analysis of sphingoid base-1-phosphates as bisacetylated derivatives by liquid chromatography-tandem mass spectrometry. Anal. Biochem. 339:129-136. http://dx.doi.org/10.1016/ j.ab.2004.12.006

Caligan, T.B., K. Peters, J. Ou, E. Wang, J. Saba, and A.H. Merrill Jr. 2000. A high-performance liquid chromatographic method to measure sphingosine 1-phosphate and related compounds from sphingosine kinase assays and other biological samples. Anal. Biochem. 281:36-44. http:// dx.doi.org/10.1006/abio.2000.4555

Chen, Z., O. Naveiras, A. Balduini, A. Mammoto, M.A. Conti, R.S. Adelstein, D. Ingber, G.Q. Daley, and R.A. Shivdasani. 2007. The May-Hegglin anomaly gene MYH9 is a negative regulator of platelet biogenesis modulated by the Rho-ROCK pathway. Blood. 110: 171-179. http://dx.doi.org/10.1182/blood-2007-02-071589

Choi, E.S., J.L. Nichol, M.M. Hokom, A.C. Hornkohl, and P. Hunt. 1995. Platelets generated in vitro from proplatelet-displaying human megakaryocytes are functional. Blood. 85:402-413.

Davis, M.D., J.J. Clemens, T.L. Macdonald, and K.R. Lynch. 2005. Sphingosine 1-phosphate analogs as receptor antagonists. J. Biol. Chem. 280:9833-9841. http://dx.doi.org/10.1074/jbc.M412356200

Dunois-Lardé, C., C. Capron, S. Fichelson, T. Bauer, E. Cramer-Bordé, and D. Baruch. 2009. Exposure of human megakaryocytes to high shear rates accelerates platelet production. Blood.114:1875-1883. http://dx.doi .org/10.1182/blood-2009-03-209205

Gérard, A., R.A. van der Kammen, H. Janssen, S.I. Ellenbroek, and J.G. Collard. 2009. The Rac activator Tiam1 controls efficient T-cell trafficking and route of transendothelial migration. Blood. 113:6138-6147. http://dx.doi.org/10.1182/blood-2008-07-167668

Golfier, S., S. Kondo, T. Schulze, T. Takeuchi, G. Vassileva, A.H. Achtman, M.H. Gräler, S.J. Abbondanzo, M. Wiekowski, E. Kremmer, et al. 2010. Shaping of terminal megakaryocyte differentiation and proplatelet development by sphingosine-1-phosphate receptor S1P4. FASEB J. 24:4701-4710. http://dx.doi.org/10.1096/fj.09-141473 
Gramaglia, I., H. Sahlin, J.P. Nolan, J.A. Frangos, M. Intaglietta, and H.C. van der Heyde. 2005. Cell- rather than antibody-mediated immunity leads to the development of profound thrombocytopenia during experimental Plasmodium berghei malaria. J. Immunol. 175:7699-7707.

Ishii, M., J.G. Egen, F. Klauschen, M. Meier-Schellersheim, Y. Saeki, J. Vacher, R.L. Proia, and R.N. Germain. 2009. Sphingosine-1-phosphate mobilizes osteoclast precursors and regulates bone homeostasis. Nature. 458:524-528. http://dx.doi.org/10.1038/nature07713

Italiano, J.E. Jr., P. Lecine, R.A. Shivdasani, and J.H. Hartwig. 1999. Blood platelets are assembled principally at the ends of proplatelet processes produced by differentiated megakaryocytes. J. Cell Biol. 147:1299-1312. http://dx.doi.org/10.1083/jcb.147.6.1299

Junt, T., H. Schulze, Z. Chen, S. Massberg, T. Goerge, A. Krueger, D.D. Wagner, T. Graf, J.E. Italiano Jr., R.A. Shivdasani, and U.H. von Andrian. 2007. Dynamic visualization of thrombopoiesis within bone marrow. Science. 317:1767-1770. http://dx.doi.org/10.1126/science.1146304

Kappos, L., J. Antel, G. Comi, X. Montalban, P. O'Connor, C.H. Polman, T. Haas, A.A. Korn, G. Karlsson, and E.W. Radue; FTY720 D2201 Study Group. 2006. Oral fingolimod (FTY720) for relapsing multiple sclerosis. N. Engl. J. Med. 355:1124-1140. http://dx.doi.org/10.1056/ NEJMoa052643

Kaushansky, K. 2005a. The molecular mechanisms that control thrombopoiesis. J. Clin. Invest. 115:3339-3347. http://dx.doi.org/10.1172/ JCI26674

Kaushansky, K. 2005b. Thrombopoietin and the hematopoietic stem cell. Ann. N. Y. Acad. Sci. 1044:139-141. http://dx.doi.org/10.1196/annals .1349 .018

Kono, M., Y. Mi, Y. Liu, T. Sasaki, M.L. Allende, Y.P. Wu, T. Yamashita, and R.L. Proia. 2004. The sphingosine-1-phosphate receptors S1P1, $\mathrm{S} 1 \mathrm{P} 2$, and S1P3 function coordinately during embryonic angiogenesis. J. Biol. Chem. 279:29367-29373. http://dx.doi.org/10.1074/jbc .M403937200

Lämmermann, T., B.L. Bader, S.J. Monkley, T. Worbs, R. WedlichSöldner, K. Hirsch, M. Keller, R. Förster, D.R. Critchley, R. Fässler, and M. Sixt. 2008. Rapid leukocyte migration by integrin-independent flowing and squeezing. Nature. 453:51-55. http://dx.doi.org/10.1038/ nature 06887

Lavenu-Bombled, C., B. Izac, F. Legrand, M. Cambot, A. Vigier, J.M. Massé, and A. Dubart-Kupperschmitt. 2007. Glycoprotein Ibalpha promoter drives megakaryocytic lineage-restricted expression after hematopoietic stem cell transduction using a self-inactivating lentiviral vector. Stem Cells. 25:1571-1577. http://dx.doi.org/10.1634/stemcells.2006-0321

Lecine, P., J.L. Villeval, P. Vyas, B. Swencki, Y. Xu, and R.A. Shivdasani. 1998. Mice lacking transcription factor NF-E2 provide in vivo validation of the proplatelet model of thrombocytopoiesis and show a platelet production defect that is intrinsic to megakaryocytes. Blood. 92: $1608-1616$.

Leeksma, C.H., and J.A. Cohen. 1955. Determination of the life of human blood platelets using labelled diisopropylfluorophosphanate. Nature. 175:552-553. http://dx.doi.org/10.1038/175552b0

Liu, Y., R. Wada, T. Yamashita, Y. Mi, C.X. Deng, J.P. Hobson, H.M. Rosenfeldt, V.E. Nava, S.S. Chae, M.J. Lee, et al. 2000. Edg-1, the G protein-coupled receptor for sphingosine-1-phosphate, is essential for vascular maturation. J. Clin. Invest. 106:951-961. http://dx.doi.org/ 10.1172/JCI10905

Majka, M., A. Janowska-Wieczorek, J. Ratajczak, M.A. Kowalska, G. Vilaire, Z.K. Pan, M. Honczarenko, L.A. Marquez, M. Poncz, and M.Z. Ratajczak. 2000. Stromal-derived factor 1 and thrombopoietin regulate distinct aspects of human megakaryopoiesis. Blood. 96:4142-4151.

Massberg, S., P. Schaerli, I. Knezevic-Maramica, M. Köllnberger, N. Tubo, E.A. Moseman, I.V. Huff, T. Junt, A.J. Wagers, I.B. Mazo, and U.H. von Andrian. 2007. Immunosurveillance by hematopoietic progenitor cells trafficking through blood, lymph, and peripheral tissues. Cell. 131:994-1008. http://dx.doi.org/10.1016/j.cell.2007.09.047

Matic, G.B., E.S. Chapman, M. Zaiss, G. Rothe, and G. Schmitz. 1998. Whole blood analysis of reticulated platelets: improvements of detection and assay stability. Cytometry. 34:229-234. http://dx.doi.org/10.1002/ (SICI) 1097-0320(19981015)34:5<229::AID-CYTO4>3.0.CO;2-2
Matloubian, M., C.G. Lo, G. Cinamon, M.J. Lesneski, Y. Xu, V. Brinkmann, M.L. Allende, R.L. Proia, and J.G. Cyster. 2004. Lymphocyte egress from thymus and peripheral lymphoid organs is dependent on S1P receptor 1. Nature. 427:355-360. http://dx.doi.org/10.1038/nature02284

Matsuyuki, H., Y. Maeda, K. Yano, K. Sugahara, K. Chiba, T. Kohno, and Y. Igarashi. 2006. Involvement of sphingosine 1-phosphate (S1P) receptor type 1 and type 4 in migratory response of mouse T cells toward S1P. Cell. Mol. Immunol. 3:429-437.

McCarty, O.J., M.K. Larson, J.M. Auger, N. Kalia, B.T. Atkinson, A.C. Pearce, S. Ruf, R.B. Henderson, V.L. Tybulewicz, L.M. Machesky, and S.P. Watson. 2005. Rac1 is essential for platelet lamellipodia formation and aggregate stability under flow. J. Biol. Chem. 280:39474-39484. http://dx.doi.org/10.1074/jbc.M504672200

Paik, J.H., A. Skoura, S.S. Chae, A.E. Cowan, D.K. Han, R.L. Proia, and T. Hla. 2004. Sphingosine 1-phosphate receptor regulation of $\mathrm{N}$-cadherin mediates vascular stabilization. Genes Dev. 18:2392-2403. http://dx.doi .org/10.1101/gad.1227804

Pang, L., H.H. Xue, G. Szalai, X. Wang, Y. Wang, D.K. Watson, W.J. Leonard, G.A. Blobel, and M. Poncz. 2006. Maturation stage-specific regulation of megakaryopoiesis by pointed-domain Ets proteins. Blood. 108:2198-2206. http://dx.doi.org/10.1182/blood-2006-04-019760

Pappu, R., S.R. Schwab, I. Cornelissen, J.P. Pereira, J.B. Regard, Y. Xu, E. Camerer, Y.W. Zheng, Y. Huang, J.G. Cyster, and S.R. Coughlin. 2007. Promotion of lymphocyte egress into blood and lymph by distinct sources of sphingosine-1-phosphate. Science. 316:295-298. http://dx.doi .org/10.1126/science.1139221

Patel, S.R., J.H. Hartwig, and J.E. Italiano Jr. 2005a. The biogenesis of platelets from megakaryocyte proplatelets. J. Clin. Invest. 115:33483354. http://dx.doi.org/10.1172/JCI26891

Patel, S.R., J.L. Richardson, H. Schulze, E. Kahle, N. Galjart, K. Drabek, R.A. Shivdasani, J.H. Hartwig, and J.E. Italiano Jr. 2005b. Differential roles of microtubule assembly and sliding in proplatelet formation by megakaryocytes. Blood. 106:4076-4085. http://dx.doi.org/10.1182/ blood-2005-06-2204

Pleines, I., A. Eckly, M. Elvers, I. Hagedorn, S. Eliautou, M. Bender, X. Wu, F. Lanza, C. Gachet, C. Brakebusch, and B. Nieswandt. 2010. Multiple alterations of platelet functions dominated by increased secretion in mice lacking Cdc42 in platelets. Blood. 115:3364-3373. http:// dx.doi.org/10.1182/blood-2009-09-242271

Pleines, I., I. Hagedorn, S. Gupta, F. May, L. Chakarova, J. van Hengel, S. Offermanns, G. Krohne, C. Kleinschnitz, C. Brakebusch, and B. Nieswandt. 2012. Megakaryocyte-specific RhoA deficiency causes macrothrombocytopenia and defective platelet activation in hemostasis and thrombosis. Blood. 119:1054-1063. http://dx.doi.org/10.1182/ blood-2011-08-372193

Radley, J.M., and C.J. Haller. 1982. The demarcation membrane system of the megakaryocyte: a misnomer? Blood. 60:213-219.

Robinson, M., S. MacHin, I. Mackie, and P. Harrison. 2000. In vivo biotinylation studies: specificity of labelling of reticulated platelets by thiazole orange and mepacrine. Br. J. Haematol. 108:859-864. http://dx.doi.org/ 10.1046/j.1365-2141.2000.01939.x

Sanna, M.G., S.K. Wang, P.J. Gonzalez-Cabrera, A. Don, D. Marsolais, M.P. Matheu, S.H. Wei, I. Parker, E. Jo, W.C. Cheng, et al. 2006 Enhancement of capillary leakage and restoration of lymphocyte egress by a chiral S1P1 antagonist in vivo. Nat. Chem. Biol. 2:434-441. http:// dx.doi.org/10.1038/nchembio804

Schulze, H., M. Korpal, J. Hurov, S.W. Kim, J. Zhang, L.C. Cantley, T. Graf, and R.A. Shivdasani. 2006. Characterization of the megakaryocyte demarcation membrane system and its role in thrombopoiesis. Blood. 107:3868-3875. http://dx.doi.org/10.1182/blood-2005-07-2755

Schwab, S.R., J.P. Pereira, M. Matloubian, Y. Xu, Y. Huang, and J.G. Cyster. 2005. Lymphocyte sequestration through S1P lyase inhibition and disruption of S1P gradients. Science. 309:1735-1739. http://dx.doi.org/ $10.1126 /$ science. 1113640

Schwer, H.D., P. Lecine, S. Tiwari, J.E. Italiano Jr., J.H. Hartwig, and R.A. Shivdasani. 2001. A lineage-restricted and divergent betatubulin isoform is essential for the biogenesis, structure and function of blood platelets. Curr. Biol. 11:579-586. http://dx.doi.org/10.1016/ S0960-9822(01)00153-1 
Spiegel, S., and S. Milstien. 2003. Sphingosine-1-phosphate: an enigmatic signalling lipid. Nat. Rev. Mol. Cell Biol. 4:397-407. http://dx.doi .org $/ 10.1038 / \mathrm{nrm} 1103$

Spiegel, S., and S. Milstien. 2011. The outs and the ins of sphingosine-1phosphate in immunity. Nat. Rev. Immunol. 11:403-415. http://dx.doi .org/10.1038/nri2974

Stenberg, P.E., and J. Levin. 1989. Mechanisms of platelet production. Blood Cells. 15:23-47.

Thon, J.N., A. Montalvo, S. Patel-Hett, M.T. Devine, J.L. Richardson, A. Ehrlicher, M.K. Larson, K. Hoffmeister, J.H. Hartwig, and J.E.
Italiano Jr. 2010. Cytoskeletal mechanics of proplatelet maturation and platelet release. J. Cell Biol. 191:861-874. http://dx.doi.org/ 10.1083/jcb. 201006102

Zengel, P.,A. Nguyen-Hoang, C. Schildhammer, R. Zantl,V. Kahl, and E. Horn. 2011. $\mu$-Slide Chemotaxis: a new chamber for long-term chemotaxis studies. BMC Cell Biol. 12:21. http://dx.doi.org/10.1186/1471-2121-12-21

Zhang, J., F.Varas, M. Stadtfeld, S. Heck, N. Faust, and T. Graf. 2007. CD41-YFP mice allow in vivo labeling of megakaryocytic cells and reveal a subset of platelets hyperreactive to thrombin stimulation. Exp. Hematol. 35:490-499. http://dx.doi.org/10.1016/j.exphem.2006.11.011 